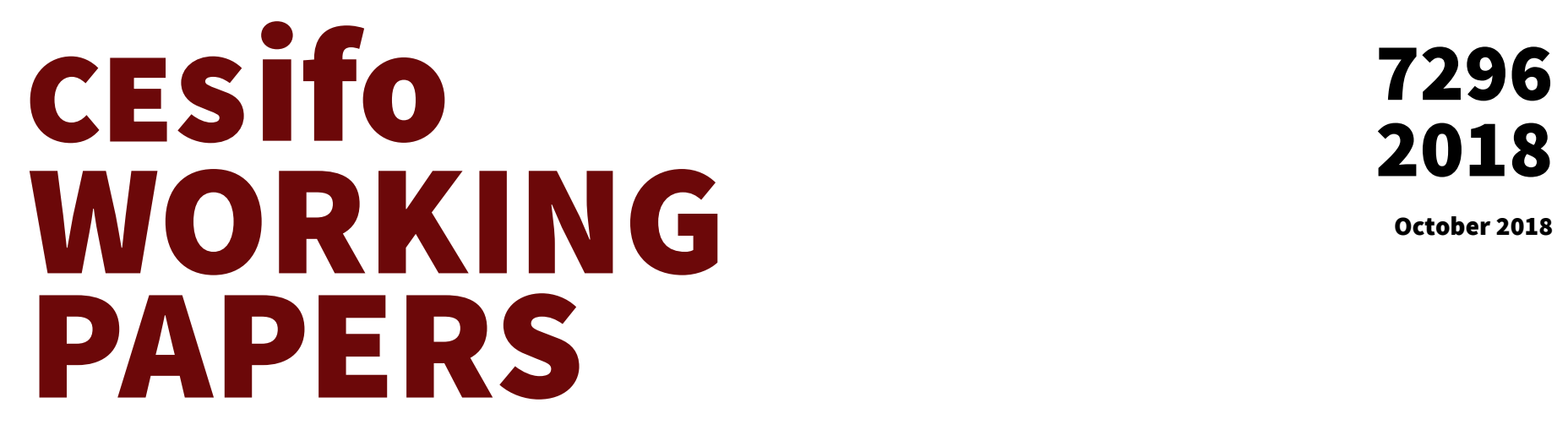

\title{
Pledge-and-Review Bargaining
}

Bård Harstad 


\section{Impressum:}

CESifo Working Papers

ISSN 2364-1428 (electronic version)

Publisher and distributor: Munich Society for the Promotion of Economic Research - CESifo $\mathrm{GmbH}$

The international platform of Ludwigs-Maximilians University's Center for Economic Studies and the ifo Institute

Poschingerstr. 5, 81679 Munich, Germany

Telephone +49 (o)89 2180-2740, Telefax +49 (o)89 2180-17845, email office@cesifo.de

Editors: Clemens Fuest, Oliver Falck, Jasmin Gröschl

www.cesifo-group.org/wp

An electronic version of the paper may be downloaded

- from the SSRN website: $\quad$ www.SSRN.com

- from the RePEc website: $\quad$ www.RePEc.org

- from the CESifo website: www.CESifo-group.org/wp 


\title{
Pledge-and-Review Bargaining
}

\begin{abstract}
Real-world negotiations differ fundamentally from existing bargaining theory. Inspired by the Paris Agreement on climate change, this paper develops a novel bargaining game in which each party quanti.es its own contribution (to a public good, for example), before the set of pledges must be accepted. I first show that, if the tolerance for delay is uncertain, each equilibrium pledge coincides with an asymmetric Nash bargaining solution. The weights placed on others. payouts reflect the underlying uncertainty, but they vary from pledge to pledge, so the set of equilibrium pledges is inefficient. This bargaining outcome is embedded in a dynamic contribution game, with endogenous technology, participation, enforcement, and contract terms, to investigate when pledge-and-review bargaining is desirable. The model's predictions can rationalize the key differences between the climate agreements signed in Kyoto (1997) and Paris (2015) as well as the development from the former to the latter.
\end{abstract}

Keywords: dynamic games, bargaining games, Nash program, climate change, Paris Agreement, Kyoto Protocol.

\author{
Bård Harstad \\ University of Oslo \\ Oslo / Norway \\ bard.harstad@econ.uio.no
}

October 1, 2018

I thank Geir Asheim, Ernesto Dal Bo, Faruk Gul, Jon Hovi, Bob Keohane, Santiago Rubio, Leo Simon, Jean Tirole, David Victor, Joel Watson, Asher Wolinsky, and audiences at UC Berkeley, CREST-Ecole Polytechnique, EIEF, ESEM 2018, University of Essex, HEC Paris, Hong Kong Baptist University, London School of Economics, Manchester University, University of Melbourne, National Taiwan University, National University of Singapore, University of Oslo, Princeton University, Queen Mary University, UC San Diego, Singapore Management University, Stanford GSB, SURED 2018, Toulouse School of Economics, and WCERE 2018. Marie Karlsen and Johannes Hveem Alsvik provided excellent research assistance and Frank Azevedo helped with the editing. 


\section{INTRODUCTION}

-The Paris talks were a bit like a potluck dinner, where guests bring what they can.

The New Yorker

December 14, 2015

Pledge-and-review $(\mathrm{P} \& \mathrm{R})$ bargaining refers to the structure of the negotiation process adopted in Paris, December 2015. Before the countries were expected to sign the climate agreement, each party was asked to submit an intended nationally determined contribution (INDC). For most developed countries, the INDC specified unconditional cuts in the emissions of greenhouse gases being effective from 2020 to 2025 (or to 2030). As an illustration, Table 1 presents the pledges for a sample of developed countries. ${ }^{1}$

\begin{tabular}{|l|c|c|c|c|c|c|c|c|}
\hline Parties & Australia & Canada & EU & New Zealand & Norway & Russia & Switzerland & USA \\
\hline Pledge & $26-28 \%$ & $30 \%$ & $40 \%$ & $30 \%$ & $40 \%$ & $25-30 \%$ & $50 \%$ & $26-28 \%$ \\
\hline
\end{tabular}

Table 1. The pledges specify emission cuts relative to nationally chosen baselines.

Every five years the parties shall review and make new pledges for another five-year period (Paris Agreement Art. 4.9).

This bargaining procedure is remarkably different from the one used for the Kyoto Protocol of 1997. There, a "top-down" approach was used to pressure governments to cut emissions by (on average) five percent relative to the 1990 levels. ${ }^{2}$ By comparison, $\mathrm{P} \& \mathrm{R}$ has been referred to as a "bottom-up" approach since countries themselves determine how much to cut nationally, without making these cuts conditional on other countries' emissions cuts. ${ }^{3}$ In political science, Keohane and Oppenheimer (2016:142) fear that: "Many governments will be tempted to use the vagueness of the Paris Agreement, and the discretion that it permits, to limit the scope or intensity of their proposed actions." Not surprisingly, leading economic theorists, such as Gollier and Tirole (2015), conclude that: "The pledge-and-review strategy is completely inadequate."

The $\mathrm{P} \& \mathrm{R}$ procedure also differs from traditional models of bargaining. To shed light on the procedure, this paper introduces and analyzes a novel bargaining game. I provide a characterization of the bargaining

\footnotetext{
${ }^{1}$ The baseline year is 1990 for the European Union, Russia, and Switzerland, while it is 2005 for Australia, Canada, New Zealand, and the United States. Article 4.4 of the Paris Agreement encourages "economy-wide absolute emission reduction targets," although several developing countries state pledges in terms of emission per GDP and some of these are conditional on receiving transfers. The official list is at http://www4.unfccc.int/ndcregistry but for an overview see http://cait.wri.org/indc/\#/.

${ }^{2}$ For example, Bodansky and Rajamani (2017:11) write: "In essence, the Kyoto Protocol was the product of mutual concessions... The USA accepted a much stronger target (minus 7\% from 1990 levels) than it had wanted..."

${ }^{3}$ According to the Paris Agreement (Art. 4.2): "Each Party shall prepare, communicate and maintain successive nationally determined contributions that it intends to achieve." So: "Now, instead of setting commitments through centralized bargaining, the Paris approach sets countries free to make their own commitments" (Victor (2015)), and: "Instead of pursuing a [Kyoto-style] top-down agreement with mandated targets, [the organizers] have asked every country to submit a national plan that lays out how and by how much they plan to reduce emissions in the years ahead" (The New York Times (Nov. 28, 2015)).
} 
outcome and relate it to the asymmetric Nash bargaining solution (ANBS). The bargaining outcome is thereafter embedded into a dynamic game where parties can both contribute to a public good and invest in their future capacity to contribute (e.g., renewable energy sources). I investigate how the bargaining procedure influences contributions, investments, participation, compliance, and payoffs.

Remarkably, the difference in bargaining procedure (Fact 1), the way I model it, can rationalize four other stylized facts on how the Paris Agreement diverges from the Kyoto Protocol: (2) While relatively few (37) countries faced binding emission targets under Kyoto, the Paris Agreement has been signed by nearly every country in the world. (3) The Kyoto Protocol was endogenously chosen in the 1990s, but the participants preferred the P\&R procedure in the 2010s. (4) While the commitments under the Kyoto Protocol were "legally binding," the INDCs are not. Despite all these differences, (5) the commitment period length agreed on was five years for both treaties. I will now explain all this in detail.

(1) The bargaining game: The novel feature of $\mathrm{P} \& \mathrm{R}$ bargaining, the way I formalize it, is that each party is permitted to propose its own individual contribution only, rather than a vector of contributions for all the parties. I assume that all parties propose their pledges simultaneously. If some parties find the vector of pledges unacceptable, there will be some delay before the procedure starts again. ${ }^{4}$ With complete information, the unique and trivial equilibrium of the game coincides with the noncooperative (or business-as-usual) outcome, where every party simply makes a pledge that maximizes that party's utility. However, with sufficiently noisy shocks on the other parties' willingness to decline and delay the agreement, I show that each party's equilibrium contribution level coincides with the quantity that maximizes an asymmetric Nash product, where the weights on other parties' payoffs reflect the extent of uncertainty as well as how the shocks are correlated. The relative weights on others' payoffs are less than $1 / 2$ for single-peaked and symmetric shock distributions, and they are close to zero when the variance of each shock is small.

Note that this bargaining game is quite general and it might be useful for several other applications beyond climate negotiations. For example, the game can describe a situation in which multiple business partners must negotiate a package, and where each partner is recognized as an expert and as the proposer for only a single dimension of the package: one partner describes the product quality, another offers a strategy for advertisements, while a third manages a set of retailers, for instance. In such expert meetings, it might be unrealistic to assume that a single partner is capable of proposing and describing all the details of interest, as is normally assumed in bargaining theory.

Although the bargaining game arguably has alternative applications, the assumptions are motivated by recent climate negotiations and thus the theory should be investigated and confronted with Facts

\footnotetext{
${ }^{4}$ The interpretation is that if $n$ sovereign countries are about to contribute, the overall agreement must be acceptable by all these $n$ countries. Before the 2009 Copenhagen negotiations, when P\&R was first attempted, many countries had submitted pledges. However: "Objections by a small group of countries (led by Bolivia, Sudan, and Venezuela) prevented the Copenhagen conference from 'adopting' the Accord"... "as a COP decision, which requires consensus (usually defined as the absence of formal objection)" (Bodansky (2010:231; 238)). In effect, negotiations were delayed for years.
} 
(2)-(5), described above. For this investigation, I present a dynamic game in which parties over time contribute to a public good (by cutting emission) as well as invest in their future capacities to contribute (e.g., they invest in renewable energy or green technology). The pledges quantify emissions targets and these are revised and updated periodically. Naturally, the small weights on others' payoffs, associated with $\mathrm{P} \& \mathrm{R}$, implies that targets are not very ambitious, and thus investments, as well as welfare, are lower than they would have been under the Nash bargaining solution (NBS), which is often used to describe the outcome of the Kyoto Protocol (see the literature review below). This negative result rationalizes the critique of the $\mathrm{P} \& \mathrm{R}$ procedure.

(2) Participation: The negative result is reversed, however, when the decision to participate in the bargaining game is endogenous. Since not much is expected from the participants (when the weights on others' payoffs are small), it is not that costly for a party to participate, and this explains why the equilibrium coalition size is larger with $\mathrm{P} \& \mathrm{R}$ bargaining than with the NBS. The larger coalition size implies that the sum of contributions is larger, the aggregate investments are larger, and so is welfare, I show.

(3) Institutional design: The comparison of bargaining procedures is more interesting when we take into account that there is a limited number $(\bar{n})$ of potential members and when this upper boundary binds. Furthermore, when the parties are heterogeneous, in that a number $(\underline{n})$ of them will participate regardless of the game, then the narrow-but-deep agreement under the NBS can be more attractive. With these constraints, $\mathrm{P} \& \mathrm{R}$ is preferred if and only if $\bar{n}$ is large while $\underline{n}$ is small, I show.

This result is in line with the development from Kyoto to Paris: In the 1990s, there were a large number of developing countries that could not be expected to contribute much to a global climate policy. Over the last twenty years, some of these have become emerging economies that potentially have important roles to play. The number of relevant potential parties, $\bar{n}$, has therefore increased. During the same period, seven countries that initially signed the Kyoto Protocol declared that they did not intend to contribute to Kyoto's second commitment period (IPCC (2014:1025)). This can be interpreted as a smaller $\underline{n}$. Either (or both) of these developments makes $\mathrm{P} \& \mathrm{R}$ more attractive. Thus, the theory can rationalize why the parties preferred the Kyoto Protocol in the 1990s, but P\&R in the 2010s.

(4) Compliance: If the parties cannot commit to future actions, the pledges must be self-enforcing. As in the repeated games literature, one may require a party to be willing to comply under the threat that others can retaliate. The P\&R bargaining outcome is more likely to be self-enforcing than the NBS, I show. The simplest intuition for this result is that when the pledges are less demanding, the temptation to defect is small. If the bargaining outcome is characterized by the NBS, in contrast, the parties might find it necessary to motivate compliance by raising the political cost of defection. In practice, the political cost can be raised by requiring the emissions cuts to be "legally binding" or enforced by other punitive measures - and both these methods are indeed employed by the Kyoto Protocol, but not by the Paris Agreement. 
(5) Terms of contract: The optimal contract duration in this model results from a novel trade-off: A long-term contract is unattractive because, after the parties have invested in new capacity, it becomes optimal to negotiate still more ambitious pledges. A short-term contract, however, creates a hold-up problem when the parties anticipate how their investments will influence the next bargaining outcome. ${ }^{5}$ The optimal term trades off these two concerns, but this trade-off is the same under P\&R as under NBS, and it is independent of the number of participants in this model. Thus, if a five-year commitment period was optimal under the Kyoto Protocol, it is indeed optimal also for the Paris Agreement, according to this result. In other words, the theory rationalizes the similarity as well as the differences between the agreements.

Literature: The $\mathrm{P} \& \mathrm{R}$ bargaining game has not been analyzed in the theoretical literature, as far as I know. By showing that each individual contribution maximizes an asymmetric Nash product, I contribute to the "Nash program," aimed at finding noncooperative games implementing cooperative solution concepts. The Nash demand game (Nash (1953)) intended to implement the NBS, axiomatized by Nash (1950). There is a large subsequent literature investigating the extent to which the Nash demand game implements the NBS: see Binmore et al. (1992), Abreu and Gul (2000), or Kambe (2000), and some contributions allow for uncertainty (Binmore (1987); Carlsson (1991); Andersson et al. (2018)). ${ }^{6}$

The alternating-offer bargaining game by Rubinstein (1982) also implements the NBS, as shown by Binmore et al. (1986). Although there can be multiple equilibria with more than two players (Sutton (1986); Osborne and Rubinstein (1990)), the NBS is the unique equilibrium if we impose stationarity or reasonable consistency conditions (Krishna and Serrano (1996); Chae and Yang (1994); Asheim (1992)). ${ }^{7}$

For these reasons, the NBS has reasonably been assumed to characterize bargaining outcomes in applied theory, such as in analyzes of climate agreements (see the surveys by Calvo and Rubio (2012) and Capparos (2016) or most of the papers discussed below). This assumption is no longer justifiable after the switch to $\mathrm{P} \& \mathrm{R}$, I show.

My contribution to this literature and to the Nash program is to show that each equilibrium pledge maximizes an asymmetric Nash product. The weights reflect differences in the discount rates in an intuitive way, but also the extent of uncertainty in shocks and the correlation in shocks across the parties. Since the equilibrium weights vary from one party's pledge to another's, the set of contributions is not Pareto optimal.

\footnotetext{
${ }^{5}$ The combined trade-off is new to the literature, but the hold-up problem associated with a small $T$ is already recognized: see, e.g., Beccherle and Tirole (2011) or Harstad (2016). Harris and Holmstrom (1987) observed that a small $T$ may be necessary to update the terms of a rigid contract when the external environment changes.

${ }^{6}$ Also the NBS with endogenous threats has been given noncooperative foundations in dynamic games: see Abreu and Pearce (2007) and (2015).

${ }^{7}$ In contrast, the ANBS (axiomatized by Harsanyi and Selten (1972); Kalai (1977); Roth (1979)) characterizes the outcome if there are asymmetric discount rates, recognition probabilities, or voting rules (Miyakawa (2008); Britz et al. (2010); Laruelle and Valenciano (2008)). There are also papers showing how the NBS is implemented in other ways, either in a specific game (Howard (1992)), in search models (Compte and Jehiel (2010)), or in a matching context (Cho and Matsui $(2013))$.
} 
The dynamic climate change game below relies on standard assumptions shared by Dutta and Radner (2004; 2006), Harstad (2012; 2016), and Battaglini and Harstad (2016), but the present paper is unique in focusing on the bargaining procedure. The coalition formation game is a standard one when modeling collusion (d'Aspremont et al. (1983); Bloch (2018)) or environmental coalitions (Hoel (1992); Carraro and Siniscalco (1993); Barrett (1994)). The resulting small coalition size is referred to as a "paradox" by Kolstad and Toman (2005) and Nordhaus (2015), but the coalition turns out to be larger with P\&R.

There is already a well-known trade-off between treaties that are narrow-but-deep vs. broad-butshallow; see Schmalensee (1998), Barrett (2002), Aldy et al. (2003), or Finus and Maus (2008). The assumptions in this literature have been criticized by political scientists such as Gilligan (2004) and Bernauer et al. (2013), but the present paper shows how the trade-off arises naturally from differences in bargaining procedures. In contrast to Schmalensee (1998), who recommended "broad, then deep," my model can rationalize the reverse, factual development from the deep-but-narrow Kyoto Protocol to the broad-but-shallow Paris Agreement.

More broadly, by showing how the bargaining procedure should be chosen to motivate participation, this paper complements the literatures on how endogenous entry influences the optimal design of mechanisms (McAfee (1993)) such as auctions (Bulow and Klemperer (1996; 2009), for example), and on how the bargaining game influences externalities and efficiency in trade negotiations (Bagwell et al. (2018)).

Outline: The next section formalizes P\&R bargaining and characterizes the outcome in Theorems 1 and 2. Section 3 embeds the bargaining outcome in a dynamic game in which parties contribute to a public good (e.g., by cutting emissions) as well as invest in their future capacities to contribute: P\&R leads to lower contributions, investments, and welfare, but this negative finding is reversed when participation is endogenous in Section 4. Section 5 argues that the theory can explain why participants have switched from preferring the NBS to preferring $\mathrm{P} \& \mathrm{R}$, while Section 6 shows that the $\mathrm{P} \& \mathrm{R}$ pledges are more likely to be self-enforcing (even if they are not legally binding). Section 7 finds that the optimal commitment period is the same for the two bargaining procedures. The robustness section presents ten extensions and explains why the results survive in all of them. Section 9 concludes. The Appendix contains all proofs.

\section{A MODEL OF PLEDGE-AND-REVIEW BARGAINING}

This section describes a novel bargaining game and characterizes its outcome. The section can be read independently from the other sections, since the model here may have alternative applications besides climate negotiations. As mentioned, the bargaining game might be appropriate when a number of business partners are negotiating a multidimensional deal, and each partner has expertise and is making the proposal on a single dimension of the package (such as quality, price, delivery time, etc.). The main new feature of the game is that each party is recognized as being responsible for proposing only one dimension of the agreement, even though payoffs depend on the entire vector. 


\subsection{The Bargaining Game}

There are $n$ parties, each endowed with a payoff function $U_{i}: \mathbb{R}^{n} \rightarrow \mathbb{R}, i \in N=\{1, \ldots n\}$. The bargaining game starts when every party $i$ simultaneously proposes its own dimension, or contribution, $x_{i} \in \mathbb{R}$, before observing the vector of proposed contributions, $\mathbf{x}=\left(x_{1}, \ldots, x_{n}\right)$. Thereafter, each party must decide whether to accept $\mathbf{x}$. If one or more parties declines $\mathbf{x}$, the game restarts after some finite delay, $\Delta \in(0, \infty)$. If everyone accepts, each party $i$ receives the payoff $U_{i}(\mathbf{x})$ and the game ends. ${ }^{8}$

I assume $U_{i}$ to be concave and continuously differentiable, and both $U_{i}$ and $x_{i}$ are measured relative to the default outcome, which thus is normalized to zero. Furthermore, I start by making the additional assumptions $\partial U_{i}(\cdot) / \partial x_{i}<(>) 0$ for $x_{i}>(<) 0$, and $\partial U_{j}(\cdot) / \partial x_{i}>0, j \neq i$, so that the $x_{i}$ 's can be interpreted as contributions to a public good. However, the Appendix proves Theorem 2 and a generalization of Theorem 1 without these additional assumptions: See the below "Remarks on Generality."

Party $j$ 's discount factor between time $t$ and $t+\Delta$ is $\delta_{j, t}^{\Delta} \leq 1$, but it will be more convenient to refer to the "discount rate" $\rho_{j, t} \equiv\left(1-\delta_{j, t}^{\Delta}\right) / \Delta .{ }^{9}$ Thus, party $j$ receives $\left(1-\rho_{j, t} \Delta\right) U_{j}\left(\mathbf{x}^{*}\right)$ by declining an offer if $\mathbf{x}^{*}$ can be expected next period. Given $\mathbf{x}^{*}, j$ prefers to accept $\mathbf{x}$ now if:

$$
U_{j}(\mathbf{x}) \geq\left(1-\rho_{j, t} \Delta\right) U_{i}\left(\mathbf{x}^{*}\right)
$$

If information were perfect, and if $\rho_{i, t}=\rho_{i}>0$ and $U_{i}(\mathbf{0}) \geq 0 \forall i \in N$, it is easy to see that a vector of proposals, $\mathbf{x}^{*}$, combined with the acceptance strategies (1), could be a stationary subgame-perfect equilibrium (SPE) only if every $x_{i}^{*}$ were equal to the noncooperative level $x_{i}^{*}=\arg \max _{x_{i}} U_{i}\left(x_{i}, \mathbf{x}_{-i}^{*}\right)$. For any other equilibrium candidate, $i$ could suggest $x_{i}$ slightly different from $x_{i}^{*}$ without violating (1). Thus, with the additional assumptions above, the "trivial equilibrium" $\mathbf{x}^{*}=\mathbf{0}$ would be unique. This observation supports the scepticism to $\mathrm{P} \& \mathrm{R}$, described in the Introduction.

In reality, a party is unlikely to know precisely the condition under which an offer will be accepted. One way of modeling this uncertainty is to assume that the exact discount rates for the next period are not known (to anyone) when the offers are made. After all, a decision maker's tolerance for delay varies with a number of temporary domestic policy or economy issues that also require the decision maker's attention. To capture this variance, write $\rho_{i, t}=\theta_{i, t} \rho_{i}$, where $\rho_{i}$ is $i$ 's expected discount rate while $\theta_{i, t}$ is a shock with mean 1 . The shocks are jointly distributed with pdf $f\left(\theta_{1, t}, \ldots, \theta_{n, t}\right) \in(0, \infty)$ on support $\prod_{i \in N}\left[0, \bar{\theta}_{i}\right]$, i.i.d. at each time $t$, and the marginal distribution of $\theta_{i, t}$ is $f_{i}\left(\theta_{i, t}\right) \equiv \int_{\Theta_{-i}} f\left(\theta_{1, t}, \ldots, \theta_{n, t}\right)$, where $\Theta_{-i} \equiv \prod_{j \neq i}\left[0, \bar{\theta}_{j}\right]$. The $\theta_{i, t}$ 's are realized and observed by everyone after the offers but before

\footnotetext{
${ }^{8}$ Two remarks are in order: (i) Unanimity is required among the parties that contribute positively, but the $n$ parties can be a subset of all players (see Sections 4 and 5). (ii) It is not assumed that the $x_{i}$ 's are enforced; only that they influence (continuation) payoffs (Section 6 discusses incentive constraints).

${ }^{9}$ If the real discount rate is $\widetilde{\rho}_{j, t}$, the discount factor is $e^{-\widetilde{\rho}_{j, t} \Delta}=\delta_{j, t}^{\Delta}$, so $\rho_{j, t} \equiv\left(1-e^{-\widetilde{\rho}_{j, t} \Delta}\right) / \Delta$ approaches $\widetilde{\rho}_{j, t}$ when $\Delta \rightarrow 0$. I refer to $\rho_{j, t}$ as the discount rate even though the identity holds only in the limit.
} 
acceptance decisions are made. ${ }^{10}$ The game is stationary, and I will henceforth restrict attention to stationary SPEs.

\subsection{The Bargaining Solution}

A stationary SPE is a vector of pledges, $\mathbf{x}^{*}$, and a set of strategies for the acceptance stage. After learning $\theta_{j, t}, j$ accepts $\mathbf{x}$ if and only if:

$$
U_{j}(\mathbf{x}) \geq\left(1-\theta_{j, t} \rho_{j} \Delta\right) U_{j}\left(\mathbf{x}^{*}\right) \Rightarrow \theta_{j, t} \geq \frac{U_{j}\left(\mathbf{x}^{*}\right)-U_{j}(\mathbf{x})}{\rho_{j} \Delta U_{j}\left(\mathbf{x}^{*}\right)} .
$$

When $\theta_{j, t}$ is drawn from a continuous distribution, the probability that $j$ accepts will be continuous in $x_{i}$. As the following result will show, this continuity can motivate larger contributions: $\mathbf{x}^{*}$ can be supported as a "nontrivial" stationary SPE if the marginal benefit for $i$ by slightly reducing $x_{i}$ is outweighed by the risk that at least one party might be sufficiently patient to decline the offer and wait for $\mathbf{x}^{*}$.

Theorem 1: Consider a nontrivial stationary SPE in which $U_{i}\left(\mathbf{x}^{*}\right)>0 \forall i$. For every $i \in N$ :

$$
\begin{aligned}
x_{i}^{*} & \leq \arg \max _{x_{i}} \prod_{j \in N}\left(U_{j}\left(x_{i}, \mathbf{x}_{-i}^{*}\right)\right)^{w_{j}^{i}}, \text { where } \\
\frac{w_{j}^{i}}{w_{i}^{i}} & =\frac{\rho_{i}}{\rho_{j}} f_{j}(0) \mathrm{E}\left(\theta_{i, t} \mid \theta_{j, t}=0\right), \forall j \neq i .
\end{aligned}
$$

Before explaining and discussing the result, note that the above reasoning does not limit how small the equilibrium $x_{i}^{*}$ 's can be, because there is no point for $i$ to contribute more than $x_{i}^{*}$, whatever the equilibrium $\mathrm{x}^{*}$ is. (The stationary equilibrium $\mathrm{x}^{*}$ will always be accepted, as is evident from (2) given that $\theta_{j, t} \geq 0$.) There can thus be multiple equilibria. To obtain sharper results, it is common to require the equilibrium to be robust to small trembles. If there is a risk that even $\mathbf{x}^{*}$ will be declined, then $i$ may prefer to reduce the risk by increasing $x_{i}$, unless (3) binds. This logic holds if we impose the following version of trembling-hand perfection. ${ }^{11}$

Definition of Local Perfection: Consider a perturbed game in which, when the vector of submitted offers is $\mathbf{x}, \mathbf{x}+\epsilon s_{t}$ is realized and observed, where $s_{t}$ is a vector of $n$ shocks distributed i.i.d. over time, with bounded support, and with strictly positive density on a neighborhood of $\mathbf{0}$. Say that $\mathbf{x}^{*}$ is locally perfect if $x_{i}^{*}=\lim _{\epsilon \rightarrow 0} x_{i}^{*}(\epsilon) \forall i \in N$, where $\mathbf{x}^{*}(\epsilon)$ is an equilibrium of the perturbed game.

\footnotetext{
${ }^{10}$ This is not unreasonable: (i) Technically, instead of letting $\Delta>0$ be the delay between rejections and new offers, $\Delta$ can be the delay between offers and acceptance decisions, if we assume that new offers can be made as soon as earlier offers are rejected. (ii) Since there is then a lag between offers and acceptance decisions, it is natural that policy makers in the meantime learn about how urgent it is for them to conclude the negotiations, or about the attention they instead must give to other policy and economic issues.

${ }^{11}$ Simon (1987) offers a similar (more general) definition when he extends to infinite games the reasoning by Myerson (1978), i.e., that the trembles in Selten (1975) should be smaller for costlier errors. Simon and Stinchcombe (1995) show that this reasoning can be adapted to infinite games in multiple ways.
} 
Theorem 2: Consider a nontrivial stationary locally perfect SPE. Inequality (3) binds for every $i \in N$.

As a comparison, in the ANBS, each $x_{i}$ maximizes the same asymmetric Nash product:

$$
x_{i}^{A}=\arg \max _{x_{i}} \prod_{j \in N}\left(U_{j}\left(x_{i}, \mathbf{x}_{-i}^{A}\right)\right)^{w_{j}},
$$

for some (exogenously) given weights. In this case, the vector $\mathbf{x}$ will be Pareto optimal.

Also when (3) binds, following P\&R bargaining, the equilibrium $x_{i}^{*}$ maximizes an asymmetric Nash product, but the weights vary with $i$ and thus the set of $x_{i}^{*}$ 's is not Pareto optimal. In particular, if $w_{j}^{i} / w_{i}^{i}<1$ for every $(i, j), j \neq i$, then it is possible to make every party better off by increasing all contributions relative to $\mathbf{x}^{*}$.

Theorem 1 also endogenizes the weights and shows how they depend on three things. First, the weight on $j$ 's utility is larger if $j$ is expected to be patient relative to $i$. This is natural (and in line with the papers on bargaining mentioned in Footnote 7): When $j$ is patient, $j$ is more tempted to reject an offer that is worse than what one can expect in the next period, and thus $i$ finds it too risky to reduce $x_{i}$, especially when $i$ is quite impatient and dislikes delay.

Second, the weight on $j$ 's payoff is larger when there is more uncertainty regarding $j$ 's shock. Of importance is especially the marginal likelihood that $j$ 's discount rate is close to 0 , so that even a small reduction from $x_{i}^{*}$ involves some risk that $j$ will decline.

Third, the weight on $j$ 's payoff is less for a small $\mathrm{E}\left(\theta_{i, t} \mid \theta_{j, t}=0\right)$, which measures $i$ 's expected shock on the discount rate given that $j$ 's shock is small. Intuitively, if $i$ can be expected to have a small discount rate exactly when $j$ has so, then it matters less that $j$ declines an offer in this circumstance. When the delay matters less, $i$ does not find it necessary to offer a lot. This suggests that a party $i$ may pay less attention to the payoffs of those who face shocks that are positively correlated with $i$ 's shock.

To conclude, each party pledges to contribute an amount that puts some weight on the utility of other parties, but only to the extent that one is uncertain about the others' willingness to accept.

\subsection{Simplifications and Generalizations}

Theorem 2 has several important consequences.

Corollary 1: Suppose all parties have the same preferences and shock distributions, $f_{i}=f_{\theta} \forall i \in N$.

(i) In a nontrivial equilibrium, $x_{i}$ is given by:

$$
\begin{aligned}
x_{i}^{*} & =\arg \max _{x_{i}} U_{i}\left(x_{i}, \mathbf{x}_{-i}^{*}\right)+w \sum_{j \neq i} U_{j}\left(x_{i}, \mathbf{x}_{-i}^{*}\right) \text {, where } \\
w & =f_{\theta}(0) E\left(\theta_{i, t} \mid \theta_{j, t}=0\right) \forall i, j .
\end{aligned}
$$




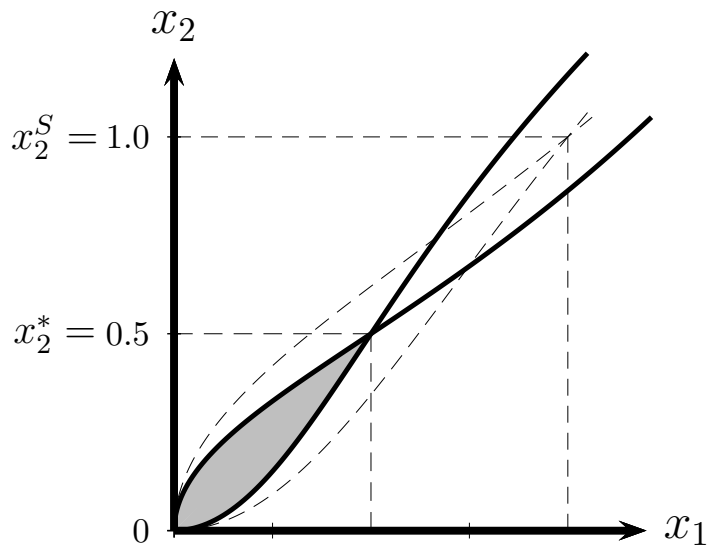

Figure 1: There are multiple equilibrium contribution levels in Example E, but they are all smaller than both the efficient level $\left(\mathbf{x}^{S}\right)$ and the locally perfect equilibrium $\left(\mathbf{x}^{*}\right)$.

(ii) The weight is $w \leq \frac{1}{2} \forall i, j \neq i$, if $f_{\theta}(\cdot)$ is single-peaked and symmetric and shocks are uncorrelated. ${ }^{12}$

Combining the two parts, the corollary suggests that the weight on other parties' payoffs is less than $\frac{1}{2}$ of the weight on $i$ 's payoff when $x_{i}$ is proposed, if the preferences are similar. If uncertainty vanishes, such that the pdf $f_{\theta}($.$) concentrates around its mean, then f_{\theta}(0) \rightarrow 0$, so $w \rightarrow 0$, and $x_{i}^{*}$ must approach the level in the trivial equilibrium, as when there is no bargaining.

Example E: As an illustration, consider the situation in which $i$ benefits linearly from the others' contributions, while each party's contribution cost is quadratic,

$$
U_{i}\left(x_{i}, \mathbf{x}_{-i}^{*}\right)=\alpha \sum_{j \neq i} x_{j}-\beta x_{i}^{2} / 2+\gamma,
$$

for some positive constants $\alpha, \beta$, and $\gamma$. Corollary 1 implies:

$$
x_{i}^{*}=w(n-1) \alpha / \beta .
$$

The grey area in Figure 1 illustrates the set of equilibria permitted by Theorem 1 when $n=2$ and $w=\frac{1}{2}$. (The pair of dashed curved lines corresponds to $w=1$, and the NBS is illustrated by $x_{1}^{S}$ and $x_{2}^{S}$.) In this example, it is easy to check that all equilibria satisfying (3) with strict inequalities are Pareto dominated by the equilibrium where the inequalities bind (i.e., $x_{1}^{*}$ and $x_{2}^{*}$ ) if just $w<\sqrt{3}-1 \approx 0,73$. Thus, focusing on equilibria that are not Pareto dominated can in some cases substitute for local perfection. ${ }^{13}$

\footnotetext{
${ }^{12}$ To see this, note that if $f_{\theta}(0)>1 / 2$, then, when $f_{\theta}(\cdot)$ is single-peaked and symmetric around the mean of one, $\int_{0}^{2} f_{\theta}\left(\theta_{j}\right) d \theta_{j}>1$, violating the definition of a pdf. If the shocks are not correlated, then $E\left(\theta_{i, t} \mid \theta_{j, t}=0\right)=1$. If the shocks are negatively correlated, then it is possible that $E\left(\theta_{i, t} \mid \theta_{j, t}=0\right)>1$.

${ }^{13}$ I thank Asher Wolinsky for making this observation.
} 
Remarks on Generality: (i) The inequality in (3) will bind for nontrivial equilibria even without requiring local perfection, if we, as an alternative, introduce trembles on the supports of the discount rates. ${ }^{14}$ (ii) Note that the theorems do not require $\Delta$ to be small. (iii) Although I assumed above that $\partial U_{i}(\cdot) / \partial x_{i}<0$ and $\partial U_{j}(\cdot) / \partial x_{i}>0, j \neq i$, these assumptions are not needed for Theorem 2 , and a generalization of Theorem 1 is proven in the Appendix without these additional assumptions.

Remark on Sufficiency: Condition (3) is necessary for $\mathbf{x}^{*}$ to be an equilibrium, but it may not be sufficient. Whether the second-order condition for an optimal deviation for $i$ holds globally depends on the pdf's $f_{j}$. If $n=2$, a sufficient condition for the second-order condition to hold is that $f_{j}$ be weakly increasing, as when $\theta_{j, t}$ is uniformly distributed, for example. ${ }^{15}$

\subsection{Remarks on Nash's Demand Game and Bargaining Solution}

The $\mathrm{P} \& \mathrm{R}$ bargaining outcome is in stark contrast to the Nash bargaining solution, predicting that the $x_{i}$ 's would follow from (4) with $w_{j}=1 \forall j$. The NBS is frequently used to describe multilateral bargaining outcomes, such as the Kyoto Protocol, partly because it results from standard noncooperative bargaining games, including the Nash demand game (NDG). Interestingly, this result follows as a corollary to Theorem 2 because $\mathrm{P} \& \mathrm{R}$ is a generalization of the NDG. ${ }^{16}$

In the NDG, each player is demanding an ex post utility level or, equivalently, a variable $\left(x_{i}\right)$ that dictates $i$ 's ex post utility, $v_{i}\left(x_{i}\right)$. The vector of demands is feasible with probability $p(\mathbf{x})$, so $i$ 's expected payoff is (see Binmore (1987) for a detailed analysis):

$$
U_{i}\left(x_{i}, \mathbf{x}_{-i}\right)=v_{i}\left(x_{i}\right) p(\mathbf{x})
$$

An alternative interpretation is that $1-p(\mathbf{x})$ is the probability for a catastrophe, eliminating all payoffs. When (5) is substituted into a binding (3), the Appendix proves:

Corollary 2: Suppose payoffs are given by (5) and every shock distribution is the same $\left(f_{i}=f_{\theta} \forall i \in\right.$ $N)$. Regardless of $f_{\theta}$, with pledge-and-review bargaining,

$$
\begin{aligned}
\mathbf{x}^{*} & =\arg \max _{\mathbf{x}} \prod_{i \in N} v_{i}\left(x_{i}\right) p(\mathbf{x})^{1+w(n-1)} \\
& =\arg \max _{\mathbf{x}} \prod_{i \in N} v_{i}\left(x_{i}\right) \text { s.t. } p(\mathbf{x})=p\left(\mathbf{x}^{*}\right) .
\end{aligned}
$$

Here, $\mathbf{x}^{*}$ coincides with the NBS in a special case, namely if the uncertainty on the feasibility constraint vanishes, in the sense that $p(\mathbf{x})$ is close to 0 or 1 for almost every $\mathbf{x}$. Then, it is intuitive that $\mathbf{x}^{*}$ must

\footnotetext{
${ }^{14}$ To be precise, Theorem 2 holds without imposing local perfection if we instead assume that the support of $\theta_{j, t}$ is $\left[\epsilon \underline{\theta}_{j}, \bar{\theta}_{j}\right]$, rather than $\left[0, \bar{\theta}_{j}\right]$, where $\underline{\theta}_{j}<0, \epsilon>0$, and $\epsilon \downarrow 0$. The interpretation of a negative discount rate may be that, in some circumstances, a party prefers to delay signing agreements because of other urgent economic/policy issues that require the decision makers' attention. It is required that the lower boundaries approach zero in the limit (as $\epsilon \rightarrow 0)$, since otherwise there will be delay on the equilibrium path.

${ }^{15}$ It is then easy to see from equation (29) in the Appendix that the second-order condition holds.

${ }^{16} \mathrm{I}$ am grateful to Jean Tirole for the motivation for this subsection.
} 
be close to an $\mathbf{x}$ that ensures $p(\mathbf{x}) \approx 1$, and so the constraint $p(\mathbf{x})=p\left(\mathbf{x}^{*}\right)$ in $(7)$ simply requires $\mathbf{x}$ to be feasible. The mapping from the NDG to the NBS is in many ways generalized by Theorem 2 and Corollary 2: The Appendix clarifies this connection and proves that, when $f$ is asymmetric, then $\mathbf{x}^{*}$ coincides with an ANBS for appropriately defined weights.

When the uncertainty on the feasibility constraint vanishes, then $U_{i}$ is discontinuous in $x_{j}$, violating the assumption in Section 2.1. If uncertainty is not vanishing, in that $p(\mathbf{x})$ is a continuous function, then (6) shows that party $i$ places less weight on the (collective) risk if $w<1$. Similarly, if the parties do not negotiate utility levels, but contribution levels, then $U_{i}(\mathbf{x})$ is likely to be continuous in all the $x_{j}$ 's, as in Example E. Given this continuity, the outcome is inefficient with $\mathrm{P} \& \mathrm{R}$ bargaining, as observed already.

\section{A DYNAMIC CONTRIBUTION GAME}

To better understand the implications of pledge-and-review, this section embeds the $\mathrm{P} \& \mathrm{R}$ bargaining solution into a tractable dynamic game. The model describes a situation in which the parties can contribute to a public good as well as invest in their future capacities to contribute. In equilibrium, the negotiated contribution levels will influence how much the parties will invest, but past investments will also influence the future contribution levels.

Although the model can be applied to other public good settings, it fits especially well to analyze climate policies. As required by the Paris Agreement (Art. 4.9): "Each Party shall communicate a nationally determined contribution every five years." Apparently, "The idea is that this short time frame would give countries the opportunity to regularly capture scientific and technological developments in their official targets." 17 The Stern Review (2006) also pointed out that new technology would be crucial to mitigate climate change. However, "technology needs must be nationally determined, based on national circumstance and priorities" ( $\$ 114$ of the 2010 Cancun Agreement). Segal (1999) provides a foundation for such contractual incompleteness, given the complexity of describing future technologies. Thus, for the model to be consistent with theory and practice, emissions cuts are negotiable and contractible, while technology investments are not. (The assumption is relaxed in Section 8.)

\subsection{The Climate Change Model}

For the dynamic model to be tractable, this section specifies linear-quadratic per-period utility functions. At each time $t$, the utility for party $i$ is the sum of three parts. First, if each party $i$ contributes or abates the quantity $q_{i, t}$, the sum of abatements has the value $a \sum_{i \in N} q_{i, t}$ to each party. This linearity assumption is made for simplicity, but it is common also because it is a reasonable approximation when

\footnotetext{
${ }^{17}$ https://www.carbonbrief.org/explainer-the-ratchet-mechanism-within-the-paris-climate-deal
} 
it comes to climate change. ${ }^{18}$ An additional benefit of this linearity is that we can easily allow for a stock of greenhouse gases that accumulates over time, without changing the analysis, since $a$ can be interpreted as the present discounted cost of emitting another unit of emission into the atmosphere, when we anticipate that this unit may contribute to climate change for decades. To see this, suppose party $i$ emits $g_{i, t}$ and the pollution stock is $G_{t}=\sigma G_{t-1}+\sum_{i \in N} g_{i, t}$, where $\sigma \in[0,1]$ measures the fraction of the past stock that survives to the next period. If parameter $h>0$ measures each party's per-period marginal environmental harm from the stock $G_{t}$, then the present discounted harm of another unit of emission is $h /(1-\sigma \delta)$ for each party. Consequently, $a \equiv h /(1-\sigma \delta)$ measures the present discounted benefit from abating a marginal unit.

The second term in the utility function specifies the cost of contributing to the public good. To fix ideas, suppose a country can consume energy from both fossil fuels $\left(g_{i, t}\right)$ and renewables $\left(R_{i, t}\right)$. If the total consumption of energy is less than $i$ 's bliss point, $B_{i, t}$, then $i$ experiences a disutility that is quadratic in the difference: $\frac{b}{2}\left(B_{i, t}-\left[g_{i, t}+R_{i, t}\right]\right)^{2}$. This disutility can be written as $\frac{b}{2}\left(q_{i, t}-R_{i, t}\right)^{2}$, when $q_{i, t}$ represents a cut in emissions relative to $i$ 's bliss point (i.e., when $q_{i, t} \equiv B_{i, t}-g_{i, t}$ ).

Of course, also for other public good situations, it will naturally be especially costly for $i$ to contribute a lot relative to $i$ 's capacity level, as represented by the stock $R_{i, t}$.

Each party can over time add to the capacity $R_{i, t}$ by investing $r_{i, t}$. The investment cost is assumed to be convex and quadratic and it constitutes the third term in the per-period utility function:

$$
\begin{aligned}
u_{i, t} & =a \sum_{j \in N} q_{j, t}-\frac{b}{2}\left(q_{i, t}-R_{i, t}\right)^{2}-\frac{c}{2} r_{i, t}^{2}, \text { where } \\
R_{i, t+1} & =R_{i, t}+r_{i, t},
\end{aligned}
$$

and where $a, b$, and $c$ are positive constants. The parties can have heterogeneous bliss points for consumption and initial technology levels $\left(R_{i, 1}\right)$. For simplicity, the parties are assumed to be identical in other respects and they plan by applying the same discount factor, $\delta .{ }^{19}$

$B A U$ : As a benchmark, consider the noncooperative Markov-perfect equilibrium (MPE) without any treaty, referred to as the "business as usual" (BAU) equilibrium. At every time $t$, when $i$ takes as given

\footnotetext{
${ }^{18}$ As Golosov et al. (2014:78) write: "Linearity is arguably not too extreme a simplification, since the composition of a concave S-to-temperature mapping with a convex temperature-to-damage function may be close to linear." They also write (p. 67), "The composition implied by Nordhaus's formulation is first concave, then convex; our function is approximately linear over this range."

${ }^{19}$ Thus, $i$ seeks to maximize $\sum_{t=0}^{\infty} \delta^{t} u_{i, t}$ at time 0 . Although a party's impatience was allowed to be stochastic and uncertain during the bargaining process in Section 2 (to make the parties' acceptance decisions uncertain), I henceforth assume the parties apply the same constant and deterministic (expected) discount factor when they decide on the longlasting investment levels. This is natural, since the uncertainty in their willingness to accept bargaining offers could be related to policy makers' need to give attention to other urgent policy or economic issues. These shocks cannot be predicted, so, for long-term technology investments, one must apply the expected discount factors (since the utility is linear in the discount factor and there is no risk aversion w.r.t. them).
} 


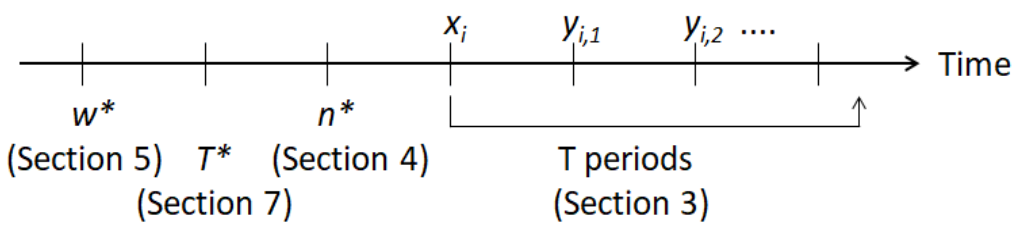

Figure 2: The timing in this and later sections (generalized in Section 8)

$R_{i, t}$, the marginal abatement cost equals the marginal benefit for party $i$ :

$$
b\left(q_{i, t}^{B A U}-R_{i, t}\right)=a \Leftrightarrow q_{i, t}^{B A U}=R_{i, t}+\frac{a}{b} .
$$

Consequently, the investment level does not influence $i$ 's future contribution cost $\left(q_{i, t}-R_{i, t}\right)$, but only $i$ 's contribution levels in every future period. Party $i$ 's preferred investment level is thus:

$$
r_{i, t}^{B A U}=\frac{\delta}{1-\delta} \frac{a}{c}
$$

With this, it is straightforward to derive party $i$ 's continuation value in BAU, $U_{i, t}^{B A U} .^{20}$

The first-best outcome is given by the exact same equations if just $a$ is replaced by na. In both cases, the second-order conditions trivially hold.

Pledges: Now, consider the possibility that the parties will contribute more than the BAU levels. In particular, suppose $i$ agrees at $t=1$ to contribute $x_{i} \geq 0$ units, beyond $i$ 's BAU level, for each of the next $T$ periods. I will continue to restrict attention to Markov-perfect strategies for the investment levels. Clearly, the commitment $x_{i}$ is payoff-relevant and it might motivate $i$ to invest $y_{i, t}$ units in addition to the BAU level. Total contributions and investments can then be written as:

$$
q_{i, t}=q_{i, t}^{B A U}+x_{i} \text { and } r_{i, t}=r_{i, t}^{B A U}+y_{i, t} .
$$

The analysis focuses on the choices of $x_{i}$ and $y_{i, t}$, since these also pin down $q_{i, t}$ and $r_{i, t}$, given BAU.

Timing: The parties negotiate the $x_{i}$ 's, pinning down the contribution levels (relative to BAU) for the next $T$ periods. Thereafter, in every period during the commitment period, each party decides on its investment level. After $T$ periods, the parties negotiate according to $\mathrm{P} \& \mathrm{R}$, once again.

After reformulating the problem, Section 3.3 discusses the equilibrium investment levels, for each point in time, as a function of the $x_{i}$ 's. This function implies that the continuation values can be summarized as a function of the $x_{i}$ 's. Thus, Theorem 2 can be applied to characterize the bargaining outcome for the $x_{i}$ 's and the effects of P\&R on equilibrium investment levels.

\footnotetext{
${ }^{20}$ As proven in the Appendix:$$
U_{i, t}^{B A U}=\frac{a}{1-\delta} \sum_{j \in N} R_{j, t}+\frac{a^{2}}{1-\delta}\left(n-\frac{1}{2}\right)\left(\frac{1}{b}+\frac{1}{c}\left[\frac{\delta}{1-\delta}\right]^{2}\right) .
$$ 
Extensions: I start by treating as exogenous parameters $n, T$, and $w$, since these could be determined by forces outside of this model, but $n$ is endogenized in Section 4, and Section 5 discusses the preference over $w$ (e.g., when P\&R bargaining is preferred to the NBS). Section 6 analyzes compliance and the conditions under which the pledges are self-enforcing, while Section 7 discusses the optimal $T$. Section 8 explains that the results also hold with timings different from the one illustrated in Figure 2 and also if pledges are functions of time, relate to investment levels or emission taxes, or if investments are made by firms rather than by governments.

\subsection{The Optimal Control Problem}

Party $i$ 's continuation value can be written as a function of the $x_{i}$ 's and the $y_{i, t}$ 's, given (9) and the BAU constants. After the pledges have been agreed on, party $i$ 's problem is to choose the investment levels over the next $T$ periods.

Lemma 1: Given the actual pledges, $\mathbf{x}$, and the future equilibrium pledges, $\mathbf{x}^{*}$, party $i$ 's continuation value is $U_{i, 1}^{B A U}+U_{i}(\mathbf{x})$, where:

$$
\begin{aligned}
U_{i}(\mathbf{x}) & \equiv \max _{\left\{y_{i, t}\right\}_{t=1}^{T}} \sum_{t=1}^{T} \delta^{t-1}\left[a \sum_{j \neq i} x_{j}-\frac{b}{2}\left(x_{i}-Y_{i, t}\right)^{2}-\frac{c}{2} y_{i, t}^{2}\right]+a \frac{\delta^{T}}{1-\delta} \sum_{j \neq i} Y_{j, T+1}+\delta^{T} U_{i}\left(\mathbf{x}^{*}\right), \\
Y_{i, t+1} & =Y_{i, t}+y_{i, t}, \text { and } Y_{i, 1}=0 .
\end{aligned}
$$

The lemma permits the current pledges $(\mathbf{x})$ to be different from those expected in equilibrium in the subsequent commitment period (i.e., $\mathbf{x}^{*}$ ). Conveniently, the heterogeneous bliss points and initial technology levels drop out when utility is measured relative to BAU.

\subsection{Equilibrium Investments}

The exact solution for the investment and the capacity levels is presented in the following lemma.

Lemma 2: For each $i \in N$ and $t \in\{1, . ., T\}$, equilibrium investment $y_{i, t}$ and $Y_{i, t}$ are linear in $x_{i}$ :

$$
\begin{aligned}
y_{i, t} & =x_{i}\left(k_{1} m_{1}^{t-1}\left[1-m_{1}\right]-k_{2} m_{2}^{t-1}\left[m_{2}-1\right]\right) \text { and } \\
Y_{i, t} & =x_{i}\left(1-k_{1} m_{1}^{t-1}-k_{2} m_{2}^{t-1}\right), \text { where } \\
m_{1} & \equiv \frac{1}{2}\left(\frac{1}{\delta}+1+\frac{b}{c}\right)-\frac{1}{2} \sqrt{\left(\frac{1}{\delta}+1+\frac{b}{c}\right)^{2}-\frac{4}{\delta}} \in(0,1), \\
m_{2} & \equiv \frac{1}{2}\left(\frac{1}{\delta}+1+\frac{b}{c}\right)+\frac{1}{2} \sqrt{\left(\frac{1}{\delta}+1+\frac{b}{c}\right)^{2}-\frac{4}{\delta}}>1, \\
k_{1} & \equiv \frac{m_{2}^{T-1}\left(m_{2}-1\right)}{m_{1}^{T-1}\left(1-m_{1}\right)+m_{2}^{T-1}\left(m_{2}-1\right)} \in(0,1), \text { and } \\
k_{2} & \equiv \frac{m_{1}^{T-1}\left(1-m_{1}\right)}{m_{1}^{T-1}\left(1-m_{1}\right)+m_{2}^{T-1}\left(m_{2}-1\right)}=1-k_{1} \in(0,1) .
\end{aligned}
$$


Naturally, if $i$ is committed to contribute a lot, in that $x_{i}$ is large, then $i$ invests more. It is also easy to check that $y_{i, t}$ decreases in both $t$ and $T$ and reaches zero when $t=T$. If the pledges are to be decided again already in the next period, then a party does not invest more than the BAU level, so the additional investment $\left(y_{i, T}\right)$ is zero. The intuition for this is the classical hold-up problem: another unit of capacity at the beginning of a new commitment period makes it possible to increase abatement by one unit, forever after, without changing the levels of investments or contribution costs. This abatement benefits everyone, not only the party that invests, just as in BAU.

\subsection{Equilibrium Contributions}

Lemma 2 stated that technology and investment levels will be linear functions of $x_{i}$. We can substitute these functions into $i$ 's utility function and write party $i$ 's continuation value (i.e., the present discounted value of the future utility levels) as a function that is quadratic in $x_{i}$. In fact, the continuation value $U_{i}(\mathbf{x})$ simplifies to Example E, introduced in Section 2, with $\alpha, \beta$ and $\gamma$ functions of $a, b, c, \delta$, and $T$ :

Lemma 3: Party i's continuation value, relative to BAU, can be written as in Example E:

$$
\begin{aligned}
U_{i}(\mathbf{x}) & =\alpha \sum_{j \neq i} x_{j}-\frac{\beta}{2} x_{i}^{2}+\gamma, \text { where } \\
\alpha & \equiv \frac{a}{1-\delta}\left[1-\delta^{T}\left(k_{1} m_{1}^{T-1}+k_{2} m_{2}^{T-1}\right)\right], \\
\beta & \equiv \sum_{t=1}^{T} \delta^{t-1}\left[b\left(k_{1} m_{1}^{t-1}+k_{2} m_{2}^{t-1}\right)^{2}+c\left(k_{1} m_{1}^{t-1}\left[1-m_{1}\right]-k_{2} m_{2}^{t-1}\left[m_{2}-1\right]\right)^{2}\right], \text { and } \\
\gamma & \equiv \delta^{T} U_{i}\left(\mathbf{x}^{*}\right) .
\end{aligned}
$$

Since the utility functions relative to BAU are symmetric, Corollary 1 implies that, with P\&R:

$$
x_{i}^{*}=\arg \max _{x_{i}}\left[U_{i}(\mathbf{x})+w \sum_{j \neq i} U_{j}(\mathbf{x})\right]=w(n-1) \alpha / \beta .
$$

The smaller $w$ is, the smaller are the $x_{i}^{*}$ 's, and the smaller are all investment levels. Both effects make the parties worse off, relative to a situation in which $w=1$. By combining (E), (11), and (10), we can see that $U_{i}\left(\mathbf{x}^{*}\right)$ increases in $w$ for every $w<1$.

Proposition 1: A smaller $w$ reduces contributions, investments, and therefore payoffs:

$$
U_{i}\left(\mathbf{x}^{*}\right)=\frac{\alpha^{2}(n-1)^{2}}{\beta\left(1-\delta^{T}\right)} w\left(1-\frac{w}{2}\right) .
$$

Fact 1: As explained in Sections 1 and 2, the Paris Agreement on climate change calls for P\&R, while the top-down negotiations associated with the Kyoto Protocol can be approximated by the NBS. Given this factual difference, Proposition 1 is consistent with the criticism mentioned in the Introduction as well as with negative experimental evidence on P\&R (Barrett and Dannenberg (2016)). The following sections show that the picture will be more nuanced when we endogenize participation. 


\section{PARTICIPATION}

This section endogenizes the coalition size and studies how it depends on the bargaining procedure. The typical way of endogenizing the coalition size is to follow the literature discussed in the Introduction. In this literature, the game begins with a participation stage at which every potential party, $i \in\{1, \ldots, \bar{n}\}$, decides whether to participate in the coalition. These decisions are made simultaneously and everyone expects that participants will continue by playing the game analyzed in Section 3. Given the restriction to MPEs, free riders will simply follow their dominant BAU strategy and set $x_{i}=0$.

It is most natural (and common) to focus on pure-strategy equilibria at the participation stage, and doing so pins down the equilibrium coalition size, $n$. I start by ignoring the constraint $n \leq \bar{n}$ as well as a possible minimum participation threshold, $\underline{n}$, but these constraints are extensively discussed in the next section. I also begin assuming that the participation decision is made once and for all, as in Figure 2, but Section 8 explains why the results continue to hold when this assumption is relaxed.

\subsection{Equilibrium Participation}

Since coalition members will contribute more than the level that would maximize their own utility, there is a cost of participating in the coalition. For a member to be willing to participate, the benefit of participating must outweigh this cost. The benefit of participating is that other participants will internalize (a fraction $w$ of) the utility of one additional coalition member.

Given Lemma 3, which states that the parties' payoffs can be summarized as (E), I henceforth restrict attention to these utility functions. The equilibrium payoff for each of the $n$ participants is given by (11).

If one of these parties instead free rides, the free rider's payoff will be $\alpha(n-1) w(n-2) \alpha / \beta\left(1-\delta^{T}\right)$, since each of the other $n-1$ parties will now contribute $w(n-2) \alpha / \beta$ every $T$ period. By comparison, participation is beneficial if:

$$
U_{i}\left(\mathbf{x}^{*}\right)=\frac{\alpha^{2}(n-1)^{2}}{\beta\left(1-\delta^{T}\right)} w\left(1-\frac{w}{2}\right) \geq \frac{\alpha^{2}(n-1)(n-2)}{\beta\left(1-\delta^{T}\right)} w \Rightarrow n \leq 1+\frac{2}{w} .
$$

The size $n$ cannot be too great since then individual contributions would be so large and so costly that free riding would be preferable. For a coalition to be stable, (12) must hold for the equilibrium $n$, but it must fail for any larger $n$ (since, otherwise, nonmembers would also like to participate). Thus, it is useful to employ the function $\lfloor$.$\rfloor , mapping its argument to the largest weakly smaller integer.$

Proposition 2: The equilibrium coalition size is decreasing in w:

$$
n=\lfloor 1+2 / w\rfloor .
$$

Note that $n=3$ if $w=1$, as when applying the NBS. This "small-coalition paradox" is well known in the literature, which also discusses the trade-off between "narrow and deep" vs. "broad and shallow" 
coalitions (see the Introduction). With $\mathrm{P} \& \mathrm{R}$ bargaining, $w$ is small and a coalition member is not expected to contribute a lot. The cost of participation is then small, and participation is attractive for a larger set of $n$ 's.

Since the number of participants must be an integer, $n$ is a step function that decreases in $w$. When comparing bargaining procedures, we are interested in large rather than small differences in $w$. Thus, it is not unreasonable to abstract from the fact that $n$ must be an integer and to use the approximation

$$
n \approx n(w) \equiv 1+2 / w .
$$

With this approximation, the product $(n(w)-1) w$ stays constant if $w$ changes. To see the intuition for this invariance, note that at the equilibrium coalition size, a party is roughly indifferent about whether to participate or free ride. On the one hand, a constant $(n-1) w$ implies that $x_{i}$ is constant and thus the cost of participation remains unchanged when $w$ changes. On the other hand, the benefit of participating is that each of the $n-1$ other parties will increase their $x_{j}$ 's by an amount proportional to $w$ if $i$ participates. This benefit is proportional to $(n-1) w$. Thus, both the benefit and the cost (and therefore the indifference condition) are unchanged when $w$ decreases if just $n$ simultaneously increases so much that the product $(n-1) w$ remains unchanged.

\subsection{Equilibrium Contributions - Revisited}

When $(n-1) w$ stays constant as $w$ is reduced, $x_{i}$ also remains constant, and so does every investment level $y_{i, t}$. Since the individual contributions are invariant in $w$, while $n$ is decreasing in $w$, overall welfare will be larger when $w$ is small. A participant's payoff is also larger when $w$ is small: this is evident when the endogenization of $n$, as described by (13), is combined with the utility (11). This gives:

$$
U_{i}^{*}=\frac{4 \alpha^{2}}{\beta\left(1-\delta^{T}\right)}\left(\frac{1}{w}-\frac{1}{2}\right)
$$

Corollary 3: With endogenous participation, approximated by $n(w)$, Proposition 1 is reversed: A smaller $w$ increases aggregate contributions, investments, and welfare.

Fact 2: While only 37 countries promised emission cuts for the Kyoto Protocol's first commitment period, 195 countries have pledged to contribute to the Paris Agreement. This fact is consistent with Proposition 2 since the Paris Agreement is associated with $\mathrm{P} \& \mathrm{R}$ and thus a smaller $w$. The popular support is the Paris Agreement's claim to success and it can reverse how we rank the two types of bargaining games, according to Corollary 3 . 


\section{WHEN TO CHOOSE PLEDGE-AND-REVIEW}

With reasonable modifications, as when $n$ is constrained, the preference concerning bargaining procedure involves a trade-off. To shed light on when pledge-and-review is preferable, this section compares participants' payoffs given a low $w=\underline{w}$ and a large $w=\bar{w}>\underline{w}$. In particular, the comparison may be between $\operatorname{NBS}(\bar{w}=1)$ and $\mathrm{P} \& \mathrm{R}(\underline{w}<1)$.

\subsection{Maximum Participation}

The world consists of a finite number $(\bar{n})$ of potential parties. If $\bar{n}<n(\bar{w})<n(\underline{w})$, where $n(\cdot)$ is defined by (13), then both bargaining games (characterized by $\underline{w}$ or $\bar{w}$ ) induce full participation. In this case, $\bar{w}$ is preferable, according to Proposition 1. If, instead, $n(\bar{w})<n(\underline{w})<\bar{n}$, the upper boundary on $n$ is nonbinding and $\underline{w}$ is preferable, according to Corollary 3. A trade-off arises when $n(\bar{w})<\bar{n}<n(\underline{w})$, since then participation is larger, but individual contributions smaller, when $w$ is small. In this case, a sufficiently large $\bar{n}$ is necessary to ensure that a participant's payoff is larger under $\underline{w}$.

The exact condition follows when comparing a participant's utility, as given by equation (11), for the two cases. The payoff is larger when $w=\underline{w}$ than when $w=\bar{w}$ if:

$$
\begin{aligned}
\frac{\alpha^{2}(\bar{n}-1)^{2}}{\beta\left(1-\delta^{T}\right)^{2}}\left(\frac{1}{\underline{w}}-\frac{1}{2}\right) & >\frac{\alpha^{2}(n(\bar{w})-1)^{2}}{\beta\left(1-\delta^{T}\right)} \bar{w}^{2}\left(\frac{1}{\bar{w}}-\frac{1}{2}\right) \Rightarrow \frac{\bar{n}-1}{n(\bar{w})-1}>\Omega, \text { where } \\
\Omega & \equiv \sqrt{\frac{\bar{w}(1-\bar{w} / 2)}{\underline{w}(1-\underline{w} / 2)}} \in\left(1, \frac{\bar{w}}{\underline{w}}\right) .
\end{aligned}
$$

\subsection{Minimum Participation}

Countries are more heterogeneous in reality than permitted in the model above. If the willingness to participate varied across countries, only some countries would be indifferent regarding whether to participate when $w$ is reduced. Countries that benefit more from participating would strictly prefer to participate, and if $w$ increased, $n$ would therefore not decline as fast as predicted by Proposition 2 .

There are several ways of capturing this reasoning in the model. Analytically, the simplest way is to assume that a number $\underline{n}$ is committed to participate regardless of $w$. The reason these parties are committed can be outside the model, but one may think of existing international treaties on non-climate issues such as international trade or regulatory politics. To be specific, European Union member countries cannot easily opt out of an environmental agreement unilaterally.

Alternatively, if the treaty specifies a minimum participation threshold for the treaty to enter into force, then $\underline{n}$ may be interpreted as this threshold. ${ }^{21}$

\footnotetext{
${ }^{21}$ In a pure-strategy equilibrium, one can correctly anticipate which countries will participate. When $n \geq \underline{n}$ binds, the $\underline{n}$ participants are here referred to as "the committed parties." These $\underline{n}$ parties must clearly agree unanimously, as assumed
} 


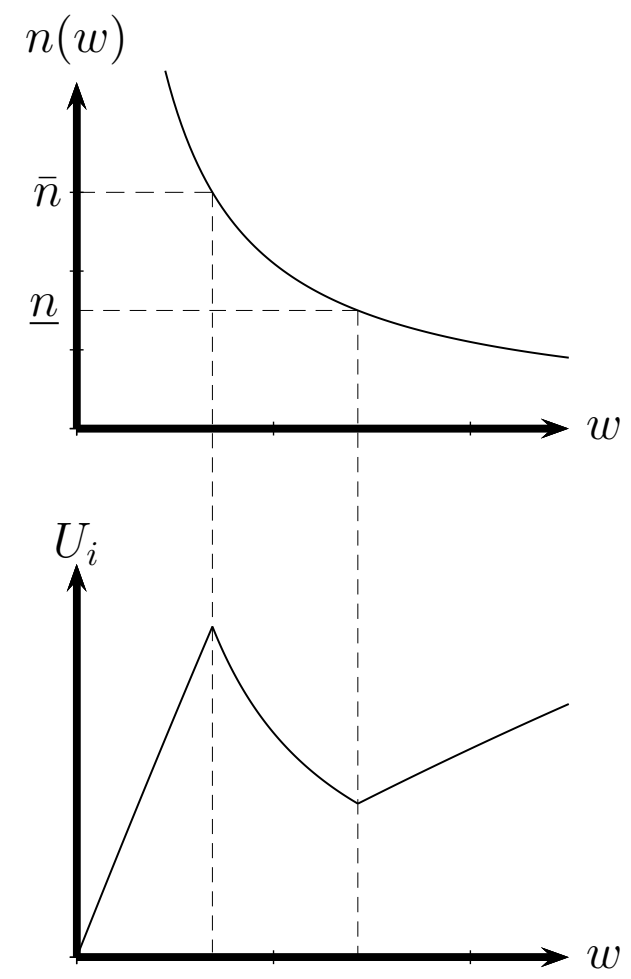

Figure 3: Participation and participants' payoffs are strictly decreasing in $w$ only when $n(w) \in(\underline{n}, \bar{n})$.

The minimum participation level $\underline{n}$ is relevant only if $\underline{n}>n(\bar{w})$. If also $\underline{n}>n(\underline{w})$, the number of participants is always $\underline{n}$ and then the larger $w$ is optimal, according to Proposition 1 . To isolate the tradeoff associated with $\underline{n}$, suppose $n(\bar{w})<\underline{n}<n(\underline{w})<\bar{n}$. In this case, only committed parties participate under $\bar{w}$, while participation under $\underline{w}$ is given by $n(\underline{w})$. By comparison, a participant's payoff can be larger under $\underline{w}$ if and only if $\underline{n}$ is sufficiently small.

The exact condition follows when we use the utility function (11) to compare the two cases. The payoff is larger when $w=\underline{w}$ than when $w=\bar{w}$ if:

$$
\frac{\alpha^{2}(n(\underline{w})-1)^{2}}{\beta\left(1-\delta^{T}\right)} \underline{w}^{2}\left(\frac{1}{\underline{w}}-\frac{1}{2}\right)>\frac{\alpha^{2}(\underline{n}-1)^{2}}{\beta\left(1-\delta^{T}\right)} \bar{w}^{2}\left(\frac{1}{\bar{w}}-\frac{1}{2}\right) \Rightarrow \frac{n(\underline{w})-1}{\underline{n}-1}>\Omega .
$$

in Section 2. Also if the equilibrium coalition size satisfies $n>\underline{n}$, the agreement must be acceptable by all $n$ contributors, if they represent sovereign countries. In this case, a subgroup of the $n$ has the option of concluding the agreement even if party $j$, say, declined $\mathbf{x}$ : But sovereign $j$ would thereafter set $x_{j}=0$, the subgroup would be worse off (the equilibrium $U_{i}\left(\mathbf{x}^{*}\right)$ increases in $\left.n\right)$, so this threat would not be credible. The alternative strategy to first negotiate and sign, then not ratify, is limited by the Vienna Convention on the Law of Treaties (Article 18), which states: "A State is obliged to refrain from acts which would defeat the object and purpose of a treaty when...it has signed the treaty..." Consequently, almost every country that signed the Kyoto Protocol (or the Paris Agreement) has also ratified; the US being the famous exception. 


\subsection{The Preferred Bargaining Game}

Figure 3 illustrates that payoffs are non-monotonic in $w$. Clearly, it is possible that both the minimum and the maximum participation levels bind at the same time. This happens if $n(\bar{w})<\underline{n}<\bar{n}<n(\underline{w})$. In this case, there is full participation under $\underline{w}$, but only the committed parties participate under $\bar{w}$. In this situation, $\underline{w}$ is preferred when $\bar{n}$ is large and $\underline{n}$ is small.

The utility function (11) shows that $\underline{w}$ gives a higher payoff than $\bar{w}$ if:

$$
\frac{\alpha^{2}(\bar{n}-1)^{2}}{\beta\left(1-\delta^{T}\right)} \underline{w}^{2}\left(\frac{1}{\underline{w}}-\frac{1}{2}\right)>\frac{\alpha^{2}(\underline{n}-1)^{2}}{\beta\left(1-\delta^{T}\right)} \bar{w}^{2}\left(\frac{1}{\bar{w}}-\frac{1}{2}\right) \Rightarrow \frac{\bar{n}-1}{\underline{n}-1}>\Omega .
$$

The three conditions can be combined in the following way.

Proposition 3: Participants prefer pledge-and-review bargaining (i.e., to switch from $w=\bar{w}$ to $w=\underline{w}<\bar{w})$ if $\bar{n}$ is large while $\underline{n}$ is small. The exact condition is:

$$
\frac{\min \{\bar{n}-1,2 / \underline{w}\}}{\max \{\underline{n}-1,2 / \bar{w}\}}>\Omega \text {. }
$$

This condition is drawn as the solid line in Figure 4. If there is a larger number of potential parties, or if fewer of them are committed to participate, we move in the direction of the arrow in the figure. Then, the "shallow" agreement $(\underline{w})$ becomes preferred even though the "deep" agreement was preferred given a smaller number of potential parties and a larger number of committed parties.

Fact 3-From Kyoto to Paris: One may argue that both these developments (i.e., a larger $\bar{n}$ and a smaller $\underline{n}$ ) are in line with changes in world politics over the last couple of decades. Today we have a large number of emerging economies which in the 1990s were developing countries that could not be expected to contribute much to an international climate change treaty. For the model, this development implies that the number of relevant parties, $\bar{n}$, has increased.

During the same period, seven of the original Annex I countries, who initially signed the Kyoto Protocol, announced that they would not contribute to the Kyoto Protocol's second commitment period. ${ }^{22}$ These withdrawals may be interpreted as a reduction in the number of committed countries, $\underline{n}$. For either reason (or both), the switch to $\mathrm{P} \& \mathrm{R}$ is consistent with the theory of this paper.

To get a sense for exactly when $\mathrm{P} \& \mathrm{R}$ is preferred, note that the Paris Agreement succeeded in motivating nearly every country in the world to participate. Given that the United States has announced that it will withdraw, it may be reasonable to assume that the weight associated with $\mathrm{P} \& \mathrm{R}$ satisfied:

$$
n(\underline{w}) \approx 195 \Leftrightarrow \underline{w} \approx \frac{1}{97} .
$$

For the sake of illustration, suppose that the Kyoto Protocol can be approximated by the NBS, with $\bar{w}=1$. When we substitute these numbers into inequality (15), Proposition 3 implies that the Paris

\footnotetext{
${ }^{22}$ According to the IPCC (2014:1025), "a number of Annex I countries (Belarus, Canada, Japan, New Zealand, Russia, the United States, and Ukraine) decided not to participate in the second commitment period."
} 


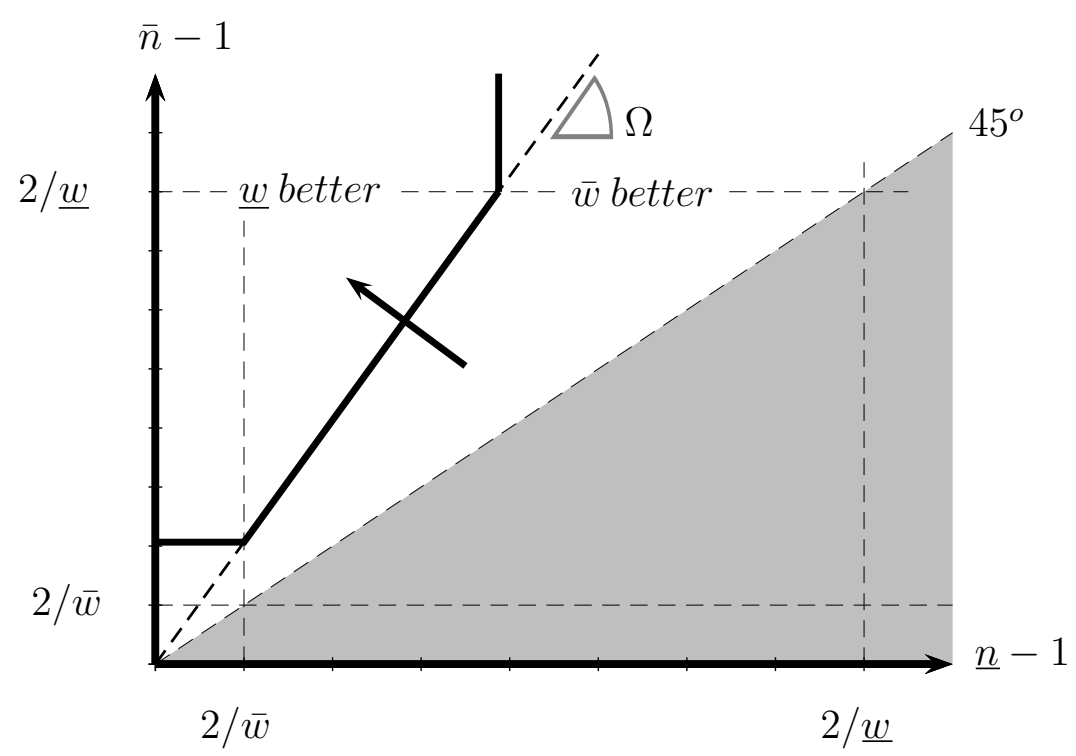

Figure 4: Participants prefer to switch to pledge-and-review ( $\underline{w})$ above the solid line.

Agreement $(\underline{w})$ is preferred to the Kyoto Protocol $(\bar{w})$ if and only if $\underline{n}$ is weakly smaller than 28 :

$$
\mathrm{P} \& \mathrm{R} \succ \mathrm{NBS} \Leftrightarrow \underline{n} \leq 28 .
$$

So, with 37 committed parties, as in the 1990s, the Kyoto Protocol is preferred. If the number of committed parties is reduced to 28 , the participants prefer $P \& R$ in order to motivate the larger set of countries to participate. Interestingly, the European Union, evidently including the most committed set of countries, consists of exactly 28 countries.

It is straightforward to show that the uncommitted countries prefer the broad agreement only when $\underline{n}<21$ in the numerical example and, in general, only when:

$$
\min \{\bar{n}-1,2 / \underline{w}\}>\sqrt{\frac{\max \{\bar{w}(\underline{n}-1) \underline{n}, 4 / \bar{w}+2\}}{\underline{w}(1-\underline{w} / 2)}},
$$

which is stronger than (15).

The theory can thus rationalize why developing countries preferred to continue with the Kyoto Protocol. Bodansky et al. (2017:202) write: "Developing countries, for which the Kyoto model has obvious attractions because they are exempt from emissions targets, were keen to extend the protocol for a second and future commitment periods. Kyoto Annex B parties, in contrast, were reluctant to do so, for some countries because of Kyoto's prescriptive architecture, and for others because they did not want to be subject to emissions targets if the US, China, and other large emitters were not."

Thus, the original set of participants prefer to switch to $\mathrm{P} \& \mathrm{R}$ too soon, that is, for a larger set of parameters than the set under which such a switch increases global welfare. Analogously, if the new potential members were pivotal in the decision on treaty design, they would accept $\mathrm{P} \& \mathrm{R}$ too late or too seldom, relative to the decision that is optimal if the original members' payoffs are taken into account. 


\section{ENFORCEMENT AND COMPLIANCE}

So far, the analysis has presumed that the pledges are contractible, credible, and complied with. Given the incentive to free ride, discussed in Sections 4 and 5 , it is reasonable to also be concerned with the temptation to contribute less at the time when other participants are expected to deliver on their promises. This section contributes to the literature on self-enforcing agreements (see, for example, Barrett (1994; 2002); Dutta and Radner (2004; 2006); Harstad et al. (2018), and the references therein) by showing when and why certain procedures, such as pledge-and-review, are more likely than others to be self-enforcing.

Since decisions are made simultaneously, a party that "defects" by not contributing will be able to enjoy the benefit from other participants' contributions in that period. Since $i$ 's cost is a convex function of $i$ 's contribution level, the temptation to defect is largest when the contribution level is large (i.e., when $w$ is large). It is thus intuitive that the incentive constraint is more likely to hold when $w$ is small, as under P\&R bargaining.

To illustrate this intuition simply, suppose the parties revert to the noncooperative MPE (BAU) forever as soon as one party has defected by contributing less than pledged. ${ }^{23}$ The analysis is particularly straightforward when investments are so costly that no one invests, since then the dynamic game boils down to a repeated game: when $c \rightarrow \infty, y_{i, t} \rightarrow 0, x_{i}^{*} \rightarrow w(n-1) a / b$, and $U_{i}\left(\mathbf{x}^{*}\right) \rightarrow \frac{(n-1)^{2}}{1-\delta} \frac{a^{2}}{b} w\left(1-\frac{w}{2}\right) \cdot{ }^{24}$ Party $i$ 's payoff when defecting is $a w(n-1)^{2} a / b$, since each of the $n-1$ other participants will contribute $w(n-1) a / b$ once, before stopping. By comparison, it is better to comply if:

$$
U_{i}\left(\mathbf{x}^{*}\right)=\frac{(n-1)^{2}}{1-\delta} \frac{a^{2}}{b} w\left(1-\frac{w}{2}\right) \geq(n-1)^{2} \frac{a^{2}}{b} w \Leftrightarrow w \leq 2 \delta .
$$

With positive investments, the dynamic game in Section 3 is different from a repeated game because past investments influence BAU and thus all future contributions. When party $j$ invests $y_{j, t}$, then $j$ 's contribution will increase by $y_{j, t}$ in every future period if the parties revert to BAU. Since every $y_{j, t}$ is largest at the beginning of the commitment period, the temptation to defect is also largest in the beginning. Then, the payoff if $i$ defects (by not contributing) is as expressed on the right-hand side in

\footnotetext{
${ }^{23} \mathrm{~A}$ few remarks are in order: The strategies to revert to BAU after a defection are not Markov. Although I here consider history-contingent strategies, it is beyond the scope of this paper to characterize the entire set of subgame-perfect equilibria. On the one hand, it is possible to sustain as SPEs harsher punishments than the reversion to BAU. With harsher punishments, a treaty would be self-enforcing under a larger set of circumstances than those derived below. On the other hand, if parties could renegotiate punishments, then a treaty would be self-enforcing for a smaller set of parameters. It is also beyond the scope of this paper to carefully analyze renegotiation-proof SPEs, but the Appendix permits the punishment to last $l \leq \infty$ periods, and to be triggered with probability $\phi \in(0,1]$. Proposition 4 holds for any $l>0$ and $\phi>0$.

${ }^{24}$ If $c \rightarrow \infty($ or $b / c \rightarrow 0)$, then $m_{1} \rightarrow 1, m_{2} \rightarrow 1 / \delta, k_{1} \rightarrow 1$, and $k_{2} \rightarrow 0$, so $y_{i, t} \rightarrow 0$ follows from Lemma 2 . Also, $\alpha \rightarrow \frac{1-\delta^{T}}{1-\delta} a$ and $\beta \rightarrow \frac{1-\delta^{T}}{1-\delta} b$, which can be substituted into (E) to derive $U_{i}\left(\mathbf{x}^{*}\right)$.
} 
the following inequality. This payoff must be smaller than $i$ 's equilibrium payoff, on the left-hand side:

$$
\begin{aligned}
U_{i}\left(\mathbf{x}^{*}\right) & =\frac{\alpha^{2}(n-1)^{2}}{\beta\left(1-\delta^{T}\right)} w\left(1-\frac{w}{2}\right) \geq a\left(\sum_{j \neq i} x_{j}+\frac{\delta}{1-\delta} \sum_{j \neq i} y_{j, 1}\right) \Leftrightarrow \\
w & \leq 2-2\left[1-\delta\left(k_{1} m_{1}+k_{2} m_{2}\right)\right] \frac{a\left(1-\delta^{T}\right)}{\alpha(1-\delta)} .
\end{aligned}
$$

The implication follows when we substitute for the equilibrium $y_{i, 1}, x_{i}$, and $\alpha$ and rewrite. The condition boils down to $w \leq 2 \delta$ when $c \rightarrow \infty$. In either case, the relevant condition is easier to satisfy when $w$ is small, i.e., if the bargaining procedure is characterized by $\mathrm{P} \& \mathrm{R}$ rather than by the NBS, for example.

Note that $n$ drops out from the inequality, and thus $n$ does not influence whether the bargaining outcome will be self-enforcing. The intuition for this invariance is that both the cost of the individual contribution and the benefit from the others' contributions are proportional to $(n-1)^{2}$. Consequently, the condition is robust to whether $n$ is exogenous (as in Section 3) or endogenous (as in Section 4).

Proposition 4: The bargaining outcome is more likely to be self-enforcing if $w$ is small. This result holds regardless of whether participation is endogenous.

Section 8 and the proof permit the punishment to last for any length $l \leq \infty$ of periods and to be triggered by any probability $\phi \in(0,1]$. For every $l>0$ and $\phi>0$, the incentive constraint will hold if and only if $w$ is sufficiently small, just as above.

If $w$ is so large that the incentive constraint is violated, then the parties must find additional ways of raising the cost of noncompliance. In reality, there are several ways of increasing these costs, since the exact wording in an international treaty influences the political and reputational costs if one later defects. Although there exists no world government ready to enforce contracts, it is not irrelevant whether a treaty is called "legally binding." IPCC (2014:1020) explains that "a more legally binding commitment ... signals a greater seriousness by states ... These factors increase the costs of violation (through enforcement and sanctions at international and domestic scales, the loss of mutual cooperation by others, and the loss of reputation and credibility in future negotiations)."

Fact 4: Since the Paris Agreement applies P\&R bargaining, where $w$ is smaller, it is more likely that the incentive constraint holds for this agreement without making it legally binding, according to Proposition 4. The pledges are indeed not legally binding under the Paris Agreement, but, as is also consistent with Proposition 4: "the Kyoto Protocol represents a much harder, more prescriptive approach, including legally binding, quantified emissions limitation targets" (Bodansky and Rajamani (2017:22)). This difference between the two agreements is consistent with Proposition 4.

Of course, when one can raise the cost of noncompliance by modifying the legal status of the agreement, then countries are likely to comply on the equilibrium path, regardless of the bargaining procedure. As predicted, even for the Kyoto Protocol, after Canada pulled out, the remaining "thirty-six Kyoto parties were in full compliance with their first commitment period targets" (Bodansky and Rajamani (2017:31)). 


\section{THE COMMITMENT PERIOD LENGTH}

The larger the length of the commitment period is, $T$, the larger the equilibrium investments are at every point in time: this can be seen from Lemma 2. This comparative static can be explained by the classic hold-up problem: if the next bargaining stage is imminent, then each party invests less because the other parties will expect larger contributions from a party that has invested in the capacity to contribute. The hold-up problem is smaller if the commitment period is longer. On the other hand, after investments have been made, it is ex post optimal for all parties to start bargaining again, to take advantage of the newly developed capacity. The participants' preferred $T$ trades off the effect on investments with the benefit that newly developed technology can strengthen the commitments sooner when $T$ is small.

Despite the trade-off when it comes to deciding on $T$, the condition for the optimal $T$ is independent of $w$ and $n$ in the model above. When $n$ is exogenous, then a party's payoff is given by equation (11). When $n$ is instead endogenous, a party's payoff is given by (14). Every participant's preferred $T$ is the same in either case:

$$
T^{*}=\arg \max _{T} U_{i}\left(\mathbf{x}^{*}\right)=\arg \max _{T} U_{i}^{*}=\arg \max _{T} \frac{\alpha^{2}}{\beta\left(1-\delta^{T}\right)},
$$

where $\alpha$ and $\beta$ are functions of $T$, as described by Lemma $3 .^{25}$

Proposition 5: The optimal commitment period length, $T^{*}$, is independent of $n$ and $w$, and of whether $n$ and $w$ are endogenous or exogenous.

Fact 5: Given the many differences between the Kyoto Protocol and the Paris Agreement, the two are surprisingly similar regarding how frequently commitments must be updated. Pledges under the Paris Agreement must be updated every five years, and the Kyoto Protocol's first commitment period was also five years (2007-2012).

It is reasonable that Kyoto's second commitment period would also have been five years, if the parties had not anticipated that a new global treaty would be effective from 2020. According to Bodansky et al. (2017:203), in 2011, "Parties disagreed on several issues including: the length of the commitment period - whether it should be five years (like the first commitment period) or eight years (to coincide with the scheduled launch of the 2015 agreement)." In 2012 (p. 205), "the eight-year duration of the second commitment period was chosen so as to end when the Paris Agreement's NDCs were expected to take effect, and thus to avoid a commitment gap."

This similarity is consistent with Proposition 5, stating that there is no reason to change the commitment period length, despite the many other differences between the two treaties. ${ }^{26}$

\footnotetext{
${ }^{25}$ If $c \rightarrow \infty$, or $b / c \rightarrow 0$, investments are irrelevant, and $\alpha^{2} / \beta\left(1-\delta^{T}\right) \rightarrow a^{2} / b(1-\delta)$, independent of $T$. In this limit, every $T$ is equally good.

${ }^{26}$ Not only the result, but also the mechanism driving it seems to match well with reality. OECD's (2018:5) first argument for a five- rather than a ten-year commitment period is: "More regular opportunities to make technical and fundamental adjustments to NDCs as well as to incorporate effects of technology..."
} 


\section{ROBUSTNESS}

The model above is simple and stylized yet able to rationalize Facts 1-5, discussed above. This rationalization is quite robust in that it continues to hold for a number of model modifications. This section explains (and the Appendix proves) that Propositions 1-5 hold even if the parties negotiate investment levels or emission taxes (or both) instead of (or in addition to) the $x_{i}$ 's. The $x_{i}$ 's can also be time dependent, and the investment levels might be decided by profit-maximizing firms, without changing the propositions. The results are also quite robust to changes in timing.

(i) Pledging to invest: Some of the INDCs in the Paris Agreement specify national targets for renewable energy. ${ }^{27}$ This possibility can be captured by letting parties decide on the $y_{i, t}$ 's instead of on the $x_{i}$ 's. As discussed in the Appendix, it is straightforward to analyze this scenario: when the $y_{i, t}$ 's, but not the $x_{i, t}$ 's, are pinned down, then $i$ 's choice of $x_{i, t}$ will satisfy $b\left(x_{i, t}-Y_{i, t}\right)=0$, just as in BAU. If the investment pledge must be time independent $\left(y_{i}\right)$ throughout a commitment period, then $i$ 's continuation value can be written as in Example (E), where $x_{i}$ is replaced by $y_{i}$, although the definitions of $\alpha$ and $\beta$ will be different. The proofs of Propositions 1-5 are thus similar to earlier proofs.

In fact, $i$ 's continuation value will be separable in the $\mathbf{y}_{t}$ 's, where $\mathbf{y}_{t}=\left(y_{1, t}, \ldots, y_{n, t}\right)$. Consequently, we can apply Corollary 1 when parties negotiate $\mathbf{y}_{t}$, while keeping fixed the investment levels for other periods. Corollary 1 will imply that the $\mathrm{P} \& \mathrm{R}$ outcome for $y_{i, t}$ is:

$$
y_{i, t}^{*}=(n-1) w \frac{\delta a / c}{1-\delta} .
$$

Since this $y_{i, t}$ is time independent, there is no loss for the parties if they restrict attention to timeindependent investment levels. For these reasons, the length of the commitment period $(T)$ will not influence payoffs, and any $T$ is here equally good, regardless of the levels of $n$ and $w$.

(ii) Pledging on emission taxes: It is also straightforward to allow parties to pledge on domestic emission taxes, instead of on emission cuts. With an emission tax $z_{i, t}$, it is natural that consumption of fossil fuel be given by the condition in which the marginal benefit of consuming (or the marginal cost of abating) equals the tax: $b\left(x_{i, t}-Y_{i, t}\right)=z_{i, t}$. When parties are free to decide their investment levels, they will invest just as in $\mathrm{BAU}$, so $y_{i, t}=0$. If the emission tax level must be time independent $\left(z_{i}\right)$ throughout the commitment period, then $i$ 's continuation value can be written as in Example (E), where $x_{i}$ is replaced by $z_{i}$, although the definitions of $\alpha$ and $\beta$ differ. Again, the proofs of Propositions 1-5 are similar to earlier proofs.

In fact, $i$ 's continuation value will be separable in the $z_{t}$ 's, where $z_{t}=\left(z_{1, t}, \ldots, z_{n, t}\right)$. Consequently, we can apply Corollary 1 when parties negotiate $z_{t}$, while keeping fixed the emission taxes for other periods.

\footnotetext{
${ }^{27}$ For example, China pledges to increase the share of non-fossil fuels in its primary energy consumption to around 20 percent, while India pledges to produce about 40 percent of its electric power from non-fossil-fuel-based energy resources by 2030. For a recent overview, see http://cait.wri.org/indc/\#/.
} 
Corollary 1 will imply that the $\mathrm{P} \& \mathrm{R}$ outcome for $z_{i, t}$ is:

$$
z_{i, t}^{*}=(n-1) w a .
$$

Since this $z_{i, t}$ is time independent, there is no loss for parties if they restrict attention to time-independent emission taxes. For these reasons, the length of the commitment period $(T)$ will not influence payoffs, and any $T$ is equally good, regardless of the levels of $n$ and $w$.

As a side remark, it is worth noting that the choice of instrument (i.e., whether parties should negotiate $x_{i}$ 's, $y_{i}$ 's, or $z_{i}$ 's) is also independent of $n$ and $w$. As proven in the Appendix, negotiating investment levels is better for all parties than negotiating emission taxes if and only if investments are inexpensive and the future is important: ${ }^{28}$

$$
\frac{1}{\delta}<1+\sqrt{\frac{b}{c}}
$$

(iii) Pledging on investment levels and emission taxes: Party $i$ 's continuation value is separable in the $\mathbf{y}_{t}$ 's and the $z_{t}$ 's, it can be shown. Thus, Corollary 1 can be applied for each instrument separately, while keeping the other fixed. With this procedure, the outcome is given by the combination of (17) and (18). In this case, we have a "complete contract" since, given the negotiated investment levels (and thus the $Y_{i, t}$ 's), the emission taxes pin down the contribution levels.

(iv) Pledging on investment levels and contribution levels: Once the investment levels (and thus the $Y_{i, t}$ 's) are pinned down, negotiating $z_{i, t}=b\left(x_{i, t}-Y_{i, t}\right)$ is equivalent to negotiating $x_{i, t}$. Thus, Scenario (iii) leads to the same outcome as that which occurs when parties can negotiate every investment level and every contribution level. As before, the choice of $T$ is irrelevant, regardless of the $n$ and $w$ levels. ${ }^{29}$

(v) Time-dependent contribution levels: In Scenario (iv), one best choice of $T$ is $T=\infty$. With $T=\infty$, it is actually irrelevant that parties have negotiated investment levels in addition to contribution levels. The irrelevance follows because, once the $x_{i, t}$ 's are given for every time, there is no externality associated with the $y_{i, t}$ 's and, hence, every party will have incentives to invest optimally, without any need to negotiate $y_{i, t}$. As is shown in the Appendix, the equilibrium time-dependent contribution level is:

$$
x_{i, t}^{*}=(n-1) w \frac{a}{b}+(n-1) w \frac{a}{c} \frac{\delta}{1-\delta} t .
$$

Given this pledge, party $i$ prefers to invest as in (17), ensuring that the marginal benefit from consuming (and from cutting emissions) is $b\left(x_{i, t}^{*}-t y_{i, t}^{*}\right)=(n-1) w a$, which coincides with $z_{i, t}^{*}$ in (18).

In this situation, it is clear that parties are strictly better off with $T=\infty$ than with $T<\infty$, since, with any finite $T$, equilibrium $y_{i, t}$ 's are lower (and less efficient) than the $y_{i, t}$ that would follow in Scenario

\footnotetext{
${ }^{28}$ The comparison to the situation in which the $x_{i}$ 's are negotiated is more complex, however.

${ }^{29}$ If parties can negotiate time-independent $x_{j}$ 's and $y_{j}$ 's, which must stay constant throughout the commitment period, then the parties would strictly prefer $T=1$. With $T=1$, the outcome will be the same as with time-dependent policies (Scenario (iv) and Scenario (iii)), while $T>1$ would be less efficient. In contrast to the discussion on the optimal $T$, in Section 7 , there is no need to have a large $T$ when the first-period investment level can be negotiated, since agreeing on $y_{i, 1}$ circumvents the hold-up problem.
} 
(iv), which coincides with the equilibrium $y_{i, t}$ 's when $T=\infty$. When referring to the trade-off discussed in the previous section, there is here no reason to reduce $T$ in order to update the pledges when the pledges can be time dependent. It is thus optimal with $T=\infty$ to mitigate the hold-up problem.

(vi) Firms invest: All three Scenarios (iii)-(v) implement the complete contract outcome, i.e., as when all $y_{i, t}$ and $x_{i, t}$ are negotiated according to P\&R. The same outcome can be achieved if parties negotiate $x_{i, t}$ at time $t$, for $T=1$, while letting firms invest. If so, the pledged $x_{i, t}$ will satisfy $b\left(x_{i, t}-Y_{i, t}\right)=$ $(n-1) w a$, which thus also characterizes the marginal willingness to pay for another unit of $Y_{i, t}$ at time $t$. Thus, the present discounted value of a unit invested today is $\delta(n-1) w a /(1-\delta)$, while the marginal investment cost is $c y_{i, t}$. The two are equalized when profit-maximizing price-taking firms decide on $y_{i, t}$ and, then, the result is (17), just as when the parties negotiate the investment levels directly.

In this situation, it is clear that parties are strictly better off with $T=1$ than with $T>1$ (unless the contribution levels are time dependent). Firms, unlike governments, are not discouraged by the nations' hold-up problem when new pledges are negotiated. ${ }^{30}$

(vii) The timing of $T$ : Proposition 5 showed that every participant agreed on the choice of $T$ and that this choice was independent of $n$ and $w$. Thus, the choice of $T$ remains the same whether participants decide on $T$ after the participation stage, before the bargaining-choice stage, or in between the two. The timing of $T$ influences neither the equilibrium level of $n$ nor the preference regarding $w$.

(viii) Multiple participation stages: Propositions 2-5 continue to hold if there is a participation stage before pledges are negotiated at the beginning of every commitment period (i.e., every $T$ period). Participating is then attractive if and only if (12) holds, just as before. The identity of the $n$ participants is also the same in every commitment period in an MPE, implying that every participant's continuation value is given by (14). Thus, the proofs of Propositions 2-5 continue to hold.

(ix) Multiple bargaining-choice stages: Propositions 2-5 also hold if $w$, as well as $n$, are endogenously chosen at the beginning of every commitment period, for the same reasons as in Scenario (viii). In fact, if some parameters (such as $\underline{n}$ and/or $\bar{n}$ ) change every $T$ period, then Propositions 2-5 characterize the outcome, and Proposition 3 characterizes the optimal bargaining procedure, for every commitment period, regardless of the parameter values after period $T$. This generalization implies that Proposition 3 can indeed rationalize a change from one procedure to another, if $\underline{n}$ and/or $\bar{n}$ has changed. ${ }^{31}$

(x) Limited punishments: When the self-enforcement constraint was discussed, Proposition 4 relied on the assumption that if one party defected, then all parties would play BAU forever after. On the one hand, one may argue that it is optimistic to assume that a defection can be observed with probability $\phi=1$. On the other hand, one may also argue that, if cooperation has broken down, then parties might renegotiate to start cooperating again. To capture these concerns to some extent, the proof of Proposition 4 permits defection to be punished with a reversion to BAU for $l \leq \infty$ periods with probability $\phi \leq 1$

\footnotetext{
${ }^{30}$ If each government can subsidize/tax the firms' investments, it can implement its preferred choice of investment, as described in the previous sections. Then, even the exact equations in Sections 3-7 stay unchanged, one can argue.

${ }^{31}$ The analysis would have been more complicated, however, if parameters changed also within commitment periods.
} 
(while, with probability $1-\phi$, there is no punishment). The incentive constraint is then:

$$
w \leq 2-2\left[\frac{1-\delta\left(k_{1} m_{1}+k_{2} m_{2}\right)}{(1-\delta)\left(1-\delta\left(\left[1-\phi+\phi \delta^{l}\right]\right)\right)}\right] \frac{a\left(1-\delta^{T}\right)}{\alpha} .
$$

Although a smaller $\phi$ or $l$ makes it more difficult to satisfy the incentive constraint, Proposition 4 continues to hold for every $\phi \in(0,1]$ and $l>0$.

These generalizations can be summarized in the following proposition (proven in the Appendix).

Proposition 6: Propositions 1-5 continue to hold if parties pledge-and-review bargain:

(i) investment levels instead of $\mathbf{x}$;

(ii) emission taxes instead of $\mathbf{x}$;

(iii) investment levels and emission taxes instead of $\mathbf{x}$;

(iv) investment levels and $\mathbf{x}$ instead of only $\mathbf{x}$;

(v) a time profile $\left\{\mathbf{x}_{t}\right\}_{t=1}^{\infty}$ instead of a time-independent $\mathbf{x}$;

(vi) $\mathbf{x}$, while profit-maximizing price-taking firms invest;

(vii) $T$ after the $n$ stage, or before $n$ but after the $w$ stage.

(viii) Propositions 2-5 continue to hold if there is a participation stage before every commitment period.

(ix) Propositions 3-5 continue to hold if both $w$ and $n$ are decided on every commitment period.

(x) Proposition 4 holds if defection leads to BAU for $l \in(0, \infty]$ periods with probability $\phi \in(0,1]$.

As discussed, the optimal level of $T$ varies across the scenarios, but for every scenario the optimal $T$ is independent of the bargaining procedure. Obviously, the optimal $T$, as well as the other results, may depend on many things that are outside of this model, such as policy makers' ability to commit to the distant future, or the ability to predict the optimal level of contributions many years in advance. Propositions 1-4 have therefore been derived for any fixed $T$, and they hold regardless of the choice of $T$.

In Harstad (2016), relying on the NBS, both pollution and shocks on the marginal environmental harm accumulate over time. The shocks make it hard to predict optimal pledges and they motivate a small $T$, while the hold-up problem motivates a large $T$, especially when there are large technological spillovers. In Battaglini and Harstad (2016), $n$ and subsequently $T$ are set endogenously before every commitment period. Then, participants may prefer a small $T$ if $n$ is small, since a small $T$ facilitates the admission of new members sooner. Since a small $T$ also leads to hold-up problems, countries are motivated to participate to ensure a large T. Acemoglu et al. (2012) permit investments in dirty as well as in green technology, Dutta and Radner (2018) transfers from the North to the South, and Martimort and Sand-Zantman (2016) a mechanism-design approach. This paper abstracts from all these extensions since the additional insights would overlap with results in the earlier papers and because these extensions are evidently not necessary to rationalize the five facts on how the Paris Agreement compares with the Kyoto Protocol. On the contrary, if the modified model did predict that $T$ should be a function of $w$ or $n$, or that any of the other propositions would change, then it would not be supported by Facts 1-5. 


\section{CONCLUDING REMARKS}

This paper presents a model and an analysis of pledge-and-review bargaining. The novelty of this bargaining game is that each party proposes how much to contribute independently - not conditional on what other parties pledge - before the parties agree to the vector of pledges. If there is some uncertainty regarding what other parties are willing to accept, for example due to shocks on the short-term discount rate, then contributions will be larger if there is a substantial variance in these shocks. In equilibrium, each party's contribution level is as described by an asymmetric Nash bargaining solution, where the weights on others' payoffs reflect the distribution and correlation of shocks. Since the weights vary from pledge to pledge, the collection of pledges is not Pareto optimal. The inefficiency may arise in business as well as political negotiations.

The P\&R bargaining game has been associated with the 2015 Paris Agreement on climate change, and it makes the agreement's approach rather different from the top-down approach that characterized the 1997 Kyoto Protocol. The analysis uncovered that (1) the factual difference in bargaining procedure can rationalize four other facts on how the Paris Agreement differs from the Kyoto Protocol: (2) Since P\&R bargaining is not very demanding, it attracts a larger number of participants. This result can explain why many more countries took on emission cuts in Paris than in Kyoto. (3) Since raising participation is the main benefit of $P \& R$, it is preferable if and only if there is a large number of relevant players. This logic can explain why P\&R was preferred in the 2010s, after several developing economies had become emerging economies, whereas the Kyoto Protocol's top-down procedure was chosen in the 1990s. (4) Since $\mathrm{P} \& \mathrm{R}$ is not very demanding, the equilibrium pledges are more likely to be self-enforcing. This result rationalizes why the Kyoto Protocol's emission cuts had to be legally binding, whereas they are not for the Paris Agreement. (5) Despite all these differences, the theory is consistent with the fact that the commitment period's length has been the same for the two agreements.

Although the paper focuses on a positive analysis, the reader may instinctively search for normative lessons. Pledge-and-review bargaining might not be as inadequate as it at first appears to be; it can actually be preferable to the alternative when participation is endogenous. However, if participation can be encouraged by other means, then a more demanding conditional-offer bargaining game becomes preferable. Consequently, the benefit of offering "club benefits" (such as the lower tariffs in Nordhaus (2015)) is not, ultimately, that participation will increase, but that parties can choose a more ambitious bargaining procedure without fearing that participation will fall by too much. 


\section{APPENDIX}

Proof of Theorem 1: As advertised in Section 2, the following generalization of Theorem 1 is here proven without the additional assumptions $\partial U_{i}(\cdot) / \partial x_{i}<0$ for $x_{i}>0$, and $\partial U_{j}(\cdot) / \partial x_{i}>0, j \neq i$.

Theorem $1^{G}$ : If $x^{*}$ is a nontrivial stationary SPE in which $U_{i}\left(\mathbf{x}^{*}\right)>0 \forall i$, then, for every $i \in N$, we have:

(a) if $\frac{\partial U_{i}\left(\mathbf{x}^{*}\right)}{\partial x_{i}} \leq 0$,

$$
-\frac{\partial U_{i}\left(\mathbf{x}^{*}\right)}{\partial x_{i}} \leq \sum_{j \neq i} \max \left\{0, \frac{\partial U_{j}\left(\mathbf{x}^{*}\right) / \partial x_{i}}{\rho_{j} \Delta U_{j}\left(\mathbf{x}^{*}\right)}\right\} f_{j}(0) \mathrm{E}\left(\theta_{i, t} \mid \theta_{j, t}=0\right) \rho_{i} \Delta U_{i}\left(\mathbf{x}^{*}\right)
$$

(b) if $\frac{\partial U_{i}\left(\mathbf{x}^{*}\right)}{\partial x_{i}}>0$,

$$
\frac{\partial U_{i}\left(\mathbf{x}^{*}\right)}{\partial x_{i}} \leq \sum_{j \neq i} \max \left\{0,-\frac{\partial U_{j}\left(\mathbf{x}^{*}\right) / \partial x_{i}}{\rho_{j} \Delta U_{j}\left(\mathbf{x}^{*}\right)}\right\} f_{j}(0) \mathrm{E}\left(\theta_{i, t} \mid \theta_{j, t}=0\right) \rho_{i} \Delta U_{i}\left(\mathbf{x}^{*}\right) .
$$

With the additional assumptions $\partial U_{i}(\cdot) / \partial x_{i}<0$ for $x_{i}>0$, and $\partial U_{j}(\cdot) / \partial x_{i}>0, j \neq i,(19)$ is equivalent to the first-order condition of (3).

(a) First, note that in any stationary SPE we must have $U_{i}\left(\mathbf{x}^{*}\right) \geq 0 \forall i$, since otherwise a party with $U_{i}\left(\mathbf{x}^{*}\right)<0$ would reject $\mathbf{x}^{*}$ in order to obtain the default payoff, normalized to zero. I will search for nontrivial equilibria in which $U_{i}\left(\mathbf{x}^{*}\right)>0 \forall i$.

A stationary equilibrium $\mathbf{x}^{*}$, such that $U_{j}\left(\mathbf{x}^{*}\right)>0 \forall j$, is accepted with probability 1 when $\rho_{j, t} \geq 0$. Therefore, $i$ will never offer $x_{i}>x_{i}^{*}$ when $\frac{\partial U_{i}\left(\mathbf{x}^{*}\right)}{\partial x_{i}} \leq 0$, so to check when $\mathbf{x}^{*}$ is an equilibrium, it is sufficient to consider a deviation by $i, \mathbf{x}^{i}$, such that $x_{i}^{i}<x_{i}^{*}$ while $x_{j}^{i}=x_{j}^{*}, j \neq i$.

Acceptable offers. Let $P\left(\mathbf{x}^{i} ; \mathbf{x}^{*}\right)$ be the probability that at least one $j \neq i$ rejects $\mathbf{x}^{i}$, and $P_{-j}\left(\mathbf{x}^{i} ; \mathbf{x}^{*}\right)$ the probability that at least one party other than $j$ and $i$ rejects $\mathbf{x}^{i}$, given an equilibrium $\mathbf{x}^{*}$.

Since party $j$ 's discount factor is $\delta_{j, t}^{\Delta} \equiv 1-\rho_{j, t} \Delta=1-\theta_{j, t} \rho_{j} \Delta, j \neq i$ rejects $\mathbf{x}^{i}$ iff:

$$
\begin{gathered}
\left(1-P_{-j}\left(\mathbf{x}^{i}\right)\right) U_{j}\left(\mathbf{x}^{i}\right)+P_{-j}\left(\mathbf{x}^{i}\right)\left(1-\rho_{j, t} \Delta\right) U_{j}\left(\mathbf{x}^{*}\right)<\left(1-\rho_{j, t} \Delta\right) U_{j}\left(\mathbf{x}^{*}\right) \Rightarrow \\
\theta_{j, t}<\widetilde{\theta}_{j}\left(\mathbf{x}^{i}\right) \equiv \max \left\{0, \frac{U_{j}\left(\mathbf{x}^{*}\right)-U_{j}\left(\mathbf{x}^{i}\right)}{\rho_{j} \Delta U_{j}\left(\mathbf{x}^{*}\right)}\right\} .
\end{gathered}
$$

When the joint pdf of shocks $\boldsymbol{\theta}_{t}=\left(\theta_{1, t}, \ldots, \theta_{n, t}\right)$ is represented by $f\left(\boldsymbol{\theta}_{t}\right)$, the probability that every $j \neq i$ accepts $\mathbf{x}^{i}$ can be written as:

$$
\begin{aligned}
1-P\left(\mathbf{x}^{i}\right) & =G\left(\widetilde{\theta}_{1}\left(\mathbf{x}^{i}\right), \ldots, \widetilde{\theta}_{i-1}\left(\mathbf{x}^{i}\right), \widetilde{\theta}_{i+1}\left(\mathbf{x}^{i}\right), \ldots \widetilde{\theta}_{n}\left(\mathbf{x}^{i}\right)\right) \\
& \equiv \int_{0}^{\bar{\theta}_{i}}\left[\int_{\tilde{\theta}_{1}\left(\mathbf{x}^{i}\right)}^{\bar{\theta}_{1}} \ldots \int_{\tilde{\theta}_{i-1}\left(\mathbf{x}^{i}\right)}^{\bar{\theta}_{i-1}} \int_{\tilde{\theta}_{i+1}\left(\mathbf{x}^{i}\right)}^{\bar{\theta}_{i+1}} \ldots \int_{\tilde{\theta}_{n}\left(\mathbf{x}^{i}\right)}^{\bar{\theta}_{n}} f\left(\boldsymbol{\theta}_{t}\right) d \boldsymbol{\theta}_{-i, t}\right] d \theta_{i},
\end{aligned}
$$

which is a function of $n-1$ thresholds (20). If we take the derivative of (21) w.r.t. $x_{i}^{i}$ and use the chain rule, we get:

$$
-\frac{\partial P\left(\mathbf{x}^{i}\right)}{\partial x_{i}}=\sum_{j \neq i}-\max \left\{0, \frac{\partial U_{j}\left(\mathbf{x}^{i}\right) / \partial x_{i}}{\rho_{j} \Delta U_{j}\left(\mathbf{x}^{*}\right)}\right\} G_{j}^{\prime}\left(\widetilde{\theta}_{1}\left(\mathbf{x}^{i}\right), \ldots, \widetilde{\theta}_{i-1}\left(\mathbf{x}^{i}\right), \widetilde{\theta}_{i+1}\left(\mathbf{x}^{i}\right), \ldots \widetilde{\theta}_{n}\left(\mathbf{x}^{i}\right)\right),
$$

and, at the equilibrium, $\mathbf{x}^{i}=\mathbf{x}^{*}$, we get:

$$
\frac{\partial P\left(\mathbf{x}^{*}\right)}{\partial x_{i}}=\sum_{j \neq i} \max \left\{0, \frac{\partial U_{j}\left(\mathbf{x}^{*}\right) / \partial x_{i}}{\rho_{j} \Delta U_{j}\left(\mathbf{x}^{*}\right)}\right\} G_{j}^{\prime}(\mathbf{0})=-\sum_{j \neq i} \max \left\{0, \frac{\partial U_{j}\left(\mathbf{x}^{*}\right) / \partial x_{i}}{\rho_{j} \Delta U_{j}\left(\mathbf{x}^{*}\right)}\right\} f_{j}(0),
$$

where, as written in the text already, $f_{j}(0)$ is the marginal distribution of $\theta_{j, t}$ at $\theta_{j, t}=0$. 
Equilibrium offers. When proposing $x_{i}$, party $i$ 's problem is to choose $x_{i} \leq x_{i}^{*}$ so as to maximize

$$
\left(1-P\left(\mathbf{x}^{i}\right)\right) U_{i}\left(\mathbf{x}^{i}\right)+P\left(\mathbf{x}^{i}\right)\left(1-\mathrm{E} \theta_{i, t}^{R} \rho_{i} \Delta\right) U_{i}\left(\mathbf{x}^{*}\right)
$$

where $\mathrm{E} \theta_{i, t}^{R}$ is the expected $\theta_{i, t}$ conditional on being rejected (this will be more precise in eq. (25)).

To derive the first-order condition w.r.t. $x_{i}^{i}$, suppose $i$ considers a small (marginal) reduction in $x_{i}$ relative to $x_{i}^{*}$, given by $d x_{i}=x_{i}^{i}-x_{i}^{*}<0$. If accepted, this gives $i$ utility

$$
U_{i}\left(\mathbf{x}^{i}\right) \approx U_{i}\left(\mathbf{x}^{*}\right)+d x_{i} \partial U_{i}\left(\mathbf{x}^{*}\right) / \partial x_{i}>U_{i}\left(\mathbf{x}^{*}\right)
$$

but $\mathbf{x}^{i}$ is rejected with probability

$$
P\left(\mathbf{x}^{i}\right) \approx P\left(\mathbf{x}^{*}\right)+\frac{\partial P\left(\mathbf{x}^{*}\right)}{\partial x_{i}} d x_{i}=0-\sum_{j \neq i} \max \left\{0, \frac{\partial U_{j}\left(\mathbf{x}^{*}\right) / \partial x_{i}}{\rho_{j} \Delta U_{j}\left(\mathbf{x}^{*}\right)}\right\} d x_{i} f_{j}(0),
$$

where each of the $n-1$ terms represents the probability that a $\theta_{j, t}$ is so small that $j$ rejects if $x_{i}$ is modified by $d x_{i}$, i.e., $\operatorname{Pr}\left(\theta_{j, t} \leq \widehat{\theta}_{j}\right)$ for $\widehat{\theta}_{j} \equiv \frac{\partial U_{j}\left(\mathbf{x}^{i}\right) / \partial x_{i}}{\rho_{j} \Delta U_{j}\left(\mathbf{x}^{*}\right)}\left|d x_{i}\right|$. Naturally, the probability that more than one party has such a small shock vanishes when $\left|d x_{i}\right| \rightarrow 0$ since $f$ is assumed to have no mass point.

If we combine (22), (23), and (24), we find party $i$ 's expected payoff when proposing $x_{i}^{i}$. This payoff, written on the left-hand side in the following inequality, is smaller than $i$ 's payoff if $i$ proposes $x_{i}^{*}$ if and only if:

$$
\begin{gathered}
\left(1-\frac{\partial P\left(\mathbf{x}^{*}\right)}{\partial x_{i}} d x_{i}\right)\left(U_{i}\left(\mathbf{x}^{*}\right)+d x_{i} \frac{\partial U_{i}\left(\mathbf{x}^{*}\right)}{\partial x_{i}}\right) \\
-\sum_{j \neq i} \max \left\{0, \frac{\partial U_{j}\left(\mathbf{x}^{*}\right) / \partial x_{i}}{\rho_{j} \Delta U_{j}\left(\mathbf{x}^{*}\right)}\right\} d x_{i} f_{j}(0)\left(1-E\left(\theta_{i, t} \mid \theta_{j, t} \leq \widehat{\theta}_{j, t}\right) \rho_{i} \Delta\right) U_{i}\left(\mathbf{x}^{*}\right) \leq U_{i}\left(\mathbf{x}^{*}\right),
\end{gathered}
$$

where $\mathrm{E}\left(\theta_{i, t} \mid \theta_{j, t} \leq \widehat{\theta}_{j}\right)$ follows from Bayes' rule:

$$
\mathrm{E}\left(\theta_{i, t} \mid \theta_{j, t} \leq \widehat{\theta}_{j}\right) \equiv \frac{\int_{0}^{\widehat{\theta}_{j}} \int_{\Theta_{-j}} \theta_{i, t} f\left(\boldsymbol{\theta}_{t}\right) d \theta_{j}}{\int_{0}^{\widehat{\theta}_{j}} \int_{\Theta_{-j}} f\left(\boldsymbol{\theta}_{t}\right) d \theta_{j}}, \text { and } \mathrm{E}\left(\theta_{i, t} \mid \theta_{j, t}=0\right) \equiv \lim _{d x_{i} \uparrow 0} \frac{\int_{0}^{\widehat{\theta}_{j}} \int_{\Theta_{-j}} \theta_{i, t} f\left(\boldsymbol{\theta}_{t}\right) d \theta_{j}}{\int_{0}^{\widehat{\theta}_{j}} \int_{\Theta_{-j}} f\left(\boldsymbol{\theta}_{t}\right) d \theta_{j}},
$$

and, as already defined, $\Theta_{-j} \equiv \prod_{k \neq j}\left[0, \bar{\theta}_{k}\right]$ and $\widehat{\theta}_{j} \equiv \frac{\partial U_{j}\left(\mathbf{x}^{*}\right) / \partial x_{i}}{\rho_{j} \Delta U_{j}\left(\mathbf{x}^{*}\right)}\left|d x_{i}\right|$.

When both sides of (25) are divided by $\left|d x_{i}\right|$ and $d x_{i} \uparrow 0,(25)$ can be rewritten as the first-order condition (19).

The proof of part (b) is analogous and thus omitted. Q.E.D.

Proof of Theorem 2: A continuum of $\mathbf{x}^{*}$ s can satisfy the equilibrium condition in Theorem $1^{G}$, since it is not necessary for $i$ to improve an offer relative to $\mathbf{x}^{*}$ when $P\left(\mathbf{x}^{*}\right)=0$. The idea of local perfection is to introduce trembles such that $P\left(\mathbf{x}^{*}\right)>0$ and thus we must check that $i$ cannot benefit from marginally increasing or decreasing $x_{i}^{i}$ from $x_{i}^{*}$ to reduce $P\left(\mathrm{x}^{i}\right)$. With trembles, $i$ will strictly benefit from $d x_{i}>0$ when (3) is strict, and thus it must hold with equality at $\mathbf{x}^{*}$.

The vector $s_{t}$ is i.i.d. over time according to some cdf, $H(\cdot)$, with is assumed to have a bounded support and $\partial H(\mathbf{0}) / \partial s_{i, t}>0$ on a neighborhood of $\mathbf{0}$. When $j$ considers whether to accept $U_{j}\left(\mathbf{x}^{i}+\epsilon \mathbf{s}_{t}\right)$, $j$ faces the continuation value $V_{j}\left(\mathbf{x}^{*}\right)$ by rejecting, where $V_{j}\left(\mathbf{x}^{*}\right)$ takes into account that $\mathbf{x}^{*}$ may be rejected in the future (if the future $s_{t}$ 's are sufficiently small):

To write the equation for $V_{j}\left(\mathbf{x}^{*}\right)$, note that it is the combination of the $s_{i, t}$ 's and the $\theta_{j, t}$ 's that determines whether $j$ rejects $\mathbf{x}^{*}$ : let $\Phi_{A}\left(\mathbf{x}^{*}\right)$ be the set of $s_{i, t}$ 's and $\theta_{j, t}$ 's such that every $j$ accepts $\mathbf{x}^{*}$, while $\Phi_{R}\left(\mathbf{x}^{*}\right)$ is the complementary set. ${ }^{32}$ We then have $P\left(\mathbf{x}^{*}\right)=\operatorname{Pr}\left\{\left(\mathbf{s}_{t}, \boldsymbol{\theta}_{t}\right) \in \Phi_{R}\left(\mathbf{x}^{*}\right)\right\}$, where $\boldsymbol{\theta}_{t}=\left(\theta_{1, t}, \ldots, \theta_{n, t}\right)$, and:

$$
V_{j}\left(\mathbf{x}^{*}\right)=\mathrm{E}_{\mathbf{s}_{t}:\left(\mathbf{s}_{t}, \boldsymbol{\theta}_{t}\right) \in \Phi_{A}\left(\mathbf{x}^{*}\right)}\left(1-P\left(\mathbf{x}^{*}\right)\right) U_{j}\left(\mathbf{x}^{*}+\epsilon \mathbf{s}_{t}\right)+P\left(\mathbf{x}^{*}\right) V_{j}\left(\mathbf{x}^{*}\right) \mathrm{E}_{\theta_{j, t}:\left(\mathbf{s}_{t}, \boldsymbol{\theta}_{t}\right) \in \Phi_{R}\left(\mathbf{x}^{*}\right)}\left(1-\theta_{j, t} \rho_{j} \Delta\right) .
$$

\footnotetext{
${ }^{32}$ By referring to $(27)$, below, $\Phi_{A}\left(\mathbf{x}^{*}\right)$ and $\Phi_{R}\left(\mathbf{x}^{*}\right)$ are defined as:
} 
The shocks, combined with the possibility to reject, imply that $V_{j}\left(\mathbf{x}^{*}\right)>0$ even if $U_{j}\left(\mathbf{x}^{*}\right)=0$, so there is no need to assume $U_{j}\left(\mathbf{x}^{*}\right)>0 \forall j$ (as was assumed for Theorem 1).

With this, party $j \neq i$ rejects $\mathbf{x}^{i}$ if and only if:

$$
\begin{gathered}
\left(1-P_{-j}\left(\mathbf{x}^{i}\right)\right) U_{j}\left(\mathbf{x}^{i}+\epsilon \mathbf{s}_{t}\right)+P_{-j}\left(\mathbf{x}^{i}\right)\left(1-\rho_{j, t} \Delta\right) V_{j}\left(\mathbf{x}^{*}\right)<\left(1-\rho_{j, t} \Delta\right) V_{j}\left(\mathbf{x}^{*}\right) \Rightarrow \\
1-\theta_{j, t} \rho_{j} \Delta>\frac{U_{j}\left(\mathbf{x}^{i}+\epsilon \mathbf{s}_{t}\right)}{V_{j}\left(\mathbf{x}^{*}\right)} \Rightarrow \theta_{j, t}<\widetilde{\theta}_{j}\left(\mathbf{x}^{i}\right) \equiv \frac{V_{j}\left(\mathbf{x}^{*}\right)-U_{j}\left(\mathbf{x}^{i}+\epsilon \mathbf{s}_{t}\right)}{\rho_{j} \Delta V_{j}\left(\mathbf{x}^{*}\right)} .
\end{gathered}
$$

Here, $\widetilde{\theta}_{j}\left(\mathbf{x}^{i}\right)$ is a function of $s_{t}$. To simplify the notation, I assume $\widetilde{\theta}_{j}\left(\mathbf{x}^{i}\right) \in\left(0, \bar{\theta}_{i}\right)$ for every $s_{t}$ when $d x_{i}<0$ is small (this is natural since we are considering small trembles when $\epsilon \rightarrow 0$ ). The probability that every $j \neq i$ accepts can then be written as:

$$
\begin{aligned}
1-P\left(\mathbf{x}^{i}\right) & =\int_{\mathbf{s}_{t}} G\left(\widetilde{\theta}_{1}\left(\mathbf{x}^{i}\right), \ldots, \widetilde{\theta}_{i-1}\left(\mathbf{x}^{i}\right), \widetilde{\theta}_{i+1}\left(\mathbf{x}^{i}\right), \ldots \widetilde{\theta}_{n}\left(\mathbf{x}^{i}\right)\right) d H\left(\mathbf{s}_{t}\right) \\
& \equiv \int_{\mathbf{s}_{t}} \int_{0}^{\bar{\theta}_{i}}\left[\int_{\widetilde{\theta}_{1}\left(\mathbf{x}^{i}\right)}^{\bar{\theta}_{1}} \ldots \int_{\widetilde{\theta}_{i-1}\left(\mathbf{x}^{i}\right)}^{\bar{\theta}_{i-1}} \int_{\tilde{\theta}_{i+1}\left(\mathbf{x}^{i}\right)}^{\bar{\theta}_{i+1}} \ldots \int_{\tilde{\theta}_{n}\left(\mathbf{x}^{i}\right)}^{\bar{\theta}_{n}} f\left(\boldsymbol{\theta}_{t}\right) d \boldsymbol{\theta}_{-i, t}\right] d \theta_{i} d H\left(\mathbf{s}_{t}\right) \Rightarrow \\
-\frac{\partial P\left(\mathbf{x}^{i}\right)}{\partial x_{i}}= & \mathrm{E}_{\mathbf{s}_{t}} \sum_{j \neq i}-\frac{\partial U_{j}\left(\mathbf{x}^{i}+\epsilon \mathbf{s}_{t}\right) / \partial x_{i}}{\rho_{j} \Delta V_{j}\left(\mathbf{x}^{*}\right)} G_{j}^{\prime}\left(\widetilde{\theta}_{1}\left(\mathbf{x}^{i}\right), \ldots, \widetilde{\theta}_{i-1}\left(\mathbf{x}^{i}\right), \widetilde{\theta}_{i+1}\left(\mathbf{x}^{i}\right), \ldots \widetilde{\theta}_{n}\left(\mathbf{x}^{i}\right)\right) .
\end{aligned}
$$

The condition under which $i$ does not benefit from a marginal change $d x_{i}<0$ is given by an equation that is similar to (25), although we now have to take into account the trembles:

$$
\begin{aligned}
& \mathrm{E}_{\mathbf{s}_{t}:\left(\mathbf{s}_{t}, \boldsymbol{\theta}_{t}\right) \in \Phi_{A}\left(\mathbf{x}^{*}\right)}\left(1-P\left(\mathbf{x}^{*}\right)-\frac{\partial P\left(\mathbf{x}^{*}\right)}{\partial x_{i}} d x_{i}\right)\left(U_{i}\left(\mathbf{x}^{*}+\epsilon \mathbf{s}_{t}\right)+\frac{\partial U_{i}\left(\mathbf{x}^{*}+\epsilon \mathbf{s}_{t}\right)}{\partial x_{i}} d x_{i}\right)+ \\
& \mathrm{E}_{\mathbf{s}_{t}} \sum_{j \neq i}\left[\frac{\partial U_{j}\left(\mathbf{x}^{*}+\epsilon \mathbf{s}_{t}\right) / \partial x_{i}}{\rho_{j} \Delta V_{j}\left(\mathbf{x}^{*}\right)} d x_{i} G_{j}^{\prime}\left(\frac{V_{1}\left(\mathbf{x}^{*}\right)-U_{1}\left(\mathbf{x}^{*}+\epsilon \mathbf{s}_{t}\right)}{\rho_{1} \Delta V_{1}\left(\mathbf{x}^{*}\right)}, \frac{V_{2}\left(\mathbf{x}^{*}\right)-U_{2}\left(\mathbf{x}^{*}+\epsilon \mathbf{s}_{t}\right)}{\rho_{2} \Delta V_{2}\left(\mathbf{x}^{*}\right)}, \ldots ; \theta_{i}\right)\right] . \\
& \mathrm{E}_{\theta_{i, t} \mid \theta_{j, t}<\widetilde{\theta}_{j}\left(\mathbf{x}^{i}\right)}\left(1-\theta_{i, t} \rho_{i} \Delta\right) V_{i}\left(\mathbf{x}^{*}\right) \leq V_{i}\left(\mathbf{x}^{*}\right) .
\end{aligned}
$$

Since the trembles imply that $P\left(\mathbf{x}^{*}\right)>0, i$ might benefit from reducing this risk and consider a marginal increase $d x_{i}>0$. Party $i$ will not benefit from $d x_{i}>0$ if (28) holds with the reverse inequality sign $(\geq)$, it is easy to show. Consequently, (28) must hold with equality for no marginal deviation to be beneficial to $i$. (Note that (28) must hold with equality regardless of whether $U_{i}(\cdot)$ would increase when $d x_{i}>0$ or when $d x_{i}<0$, so, we do not need the assumptions $\partial U_{i}(\cdot) / \partial x_{j}>0$ for $j \neq i$ and $<0$ for $j=i$.)

When we let $\epsilon \rightarrow 0$, so that the trembles vanish, then we can see from (26) and (27) that $P\left(\mathbf{x}^{*}\right) \rightarrow 0$ and $V_{j}\left(\mathbf{x}^{*}\right) \rightarrow U_{j}\left(\mathbf{x}^{*}\right)$. When these limits are substituted into (28), holding with equality, and we divide both sides by $d x_{i}$ before we let $d x_{i} \rightarrow 0$ and $\epsilon s_{t} \rightarrow 0$, then (28) can be rewritten as:

$$
\frac{\partial U_{i}\left(\mathbf{x}^{*}\right)}{\partial x_{i}}+\sum_{j \neq i} \frac{\partial U_{j}\left(\mathbf{x}^{*}\right) / \partial x_{i}}{\rho_{j} \Delta U_{j}\left(\mathbf{x}^{*}\right)} f_{j}(0) \mathrm{E}\left(\theta_{i, t} \mid \theta_{j, t}=0\right) \rho_{i} \Delta U_{i}\left(\mathbf{x}^{*}\right)=0,
$$

which coincides with the first-order condition of

$$
\arg \max _{x_{i}} \prod_{j \in N}\left(U_{j}\left(x_{i}, \mathbf{x}_{-i}^{*}\right)\right)^{w_{j}^{i}}
$$

when $\frac{w_{j}^{i}}{w_{i}^{i}}=\frac{\rho_{i}}{\rho_{j}} f_{j}(0) \mathrm{E}\left(\theta_{i, t} \mid \theta_{j, t}=0\right), \forall j \neq i$. Q.E.D.

$$
\begin{aligned}
\Phi_{A}\left(\mathbf{x}^{*}\right) & =\left\{\left(\mathbf{s}_{t}, \boldsymbol{\theta}_{t}\right): \theta_{j, t} \geq \frac{V_{j}\left(\mathbf{x}^{*}\right)-U_{j}\left(\mathbf{x}^{*}+\epsilon \mathbf{s}_{t}\right)}{\rho_{j} \Delta V_{j}\left(\mathbf{x}^{*}\right)} \forall j\right\} \\
\Phi_{R}\left(\mathbf{x}^{*}\right) & =\left\{\left(\mathbf{s}_{t}, \boldsymbol{\theta}_{t}\right): \theta_{j, t}<\frac{V_{j}\left(\mathbf{x}^{*}\right)-U_{j}\left(\mathbf{x}^{*}+\epsilon \mathbf{s}_{t}\right)}{\rho_{j} \Delta V_{j}\left(\mathbf{x}^{*}\right)} \text { for at least one } j\right\} .
\end{aligned}
$$


Proof of Corollary 2 and the Nash Demand Game: I start by presenting a more general result.

Theorem 3: Suppose every payoff is given by (5). With pledge-and-review bargaining,

$$
\begin{aligned}
\mathbf{x}^{*}= & \arg \max _{\mathbf{x}} \prod_{i \in N} v_{i}\left(x_{i}\right)^{\varrho_{i}} p(\mathbf{x})^{\varpi} \\
= & \arg \max _{\mathbf{x}} \prod_{i \in N} v_{i}\left(x_{i}\right)^{\varrho_{i}} \text { s.t. } p(\mathbf{x})=p\left(\mathbf{x}^{*}\right) \text {, where } \\
& \varrho_{i}=\frac{w_{i}^{i} / \sum_{j \in N} w_{j}^{i}}{\sum_{k \in N}\left(w_{k}^{k} / \sum_{j \in N} w_{j}^{k}\right)} \text { and } \varpi=\frac{1}{\sum_{k \in N}\left(w_{k}^{k} / \sum_{j \in N} w_{j}^{k}\right)} .
\end{aligned}
$$

Proof: With (5), a binding (3) implies:

$$
\begin{aligned}
x_{i}^{*} & =\arg \max _{x_{i}} \prod_{j \in N}\left(v_{j}\left(x_{j}\right) p(\mathbf{x})\right)^{w_{j}^{i}}=\arg \max _{x_{i}} v_{i}\left(x_{i}\right) p(\mathbf{x})^{\sum_{j} w_{j}^{i} / w_{i}^{i}} \\
& =\arg \max _{x_{i}} v_{i}\left(x_{i}\right)^{w_{i}^{i} / \sum_{j} w_{j}^{i}} p(\mathbf{x})=\arg \max _{x_{i}} \prod_{j \in N} v_{j}\left(x_{j}\right)^{w_{j}^{j} / \sum_{k} w_{k}^{j}} p(\mathbf{x}), \text { so } \\
\mathbf{x}^{*} & =\arg \max _{\mathbf{x}} \prod_{j \in N} v_{j}\left(x_{j}\right)^{w_{j}^{j} / \sum_{k} w_{k}^{j}} p(\mathbf{x}),
\end{aligned}
$$

which can be written as (30), given the definitions $\varrho_{i}$ and $\omega$. Given $\mathbf{x}^{*},(30)$ can be rewritten as (31).

The theorem basically follows as a corollary to Theorem 2. Corollary 2 follows, in turn, when we simplify with $w_{j}^{i} / w_{i}^{i}=w \forall j \neq i$. Q.E.D.

Discussion on the relationship to the NDG: The outome, (31), coincides with the NBS if the uncertainty on the feasibility constraint vanishes, and if the weights $\left(w_{j}^{i} / w_{i}^{i}\right)$ are equal. The weights are equal holds if, for example, as in the traditional literature, $f_{i}(0)=0 \forall i \in N$ (then, $\varrho_{i}=\varpi=1$ ). In the traditional NDG, there is neither voting nor additional rounds, so party $i$ does not need to fear that the others will reject the proposal, just as in the P\&R game when $f_{i}(0)=0 \forall i$.

The mapping from the NDG to the NBS is in several respects generalized by Theorem 3 :

(i) Theorem 3 confirms that the mapping holds if there is a finite delay when $\mathbf{x}$ is not approved unanimously, before the game can be played again.

(ii) The theorem also permits stochastic discount rates and shows that these are irrelevant for the mapping if parties are symmetric.

Despite these differences to the traditional NDG, the outcome continues to be the NBS if the parties are similar, regardless of the level of the common $w$. The intuition for why $w$ is irrelevant is that, given the sharp threshold characterized by $p(\mathbf{x})$ when uncertainty vanishes, $i$ 's preferred $x_{i}$ coincides with the efficient level, given the other $x_{j}$ 's.

(iii) When the weights $\left(w_{j}^{i} / w_{i}^{i}\right)$ are heterogeneous, the outcome (31) is given by an ANBS: The bargaining power index $\left(\varrho_{i}\right)$ is larger for those parties who are likely to be patient or who face less uncertainty regarding the opponents' discount rates, it can be shown.

(vi) More fundamentally, if each $U_{i}(\mathbf{x})$ is continuous in every $x_{j}$, as when the uncertainty on the feasibility constraint is not vanishing, then, with $\mathrm{P} \& \mathrm{R}$ bargaining, $\mathrm{x}^{*}$ does not coincide with any ANBS. Instead, each $x_{i}^{*}$, given $\mathbf{x}_{-i}^{*}$, coincides with an ANBS where the weights depend on $i$.

Proof of Lemma 1: I will first derive $U_{i, t}^{B A U}$. When we substitute in for $u_{i, t}, q_{i, t}^{B A U}$, and $r_{i, t}^{B A U}$ into 


$$
\begin{aligned}
& U_{i, t}^{B A U}=\sum_{\tau=t}^{\infty} \delta^{\tau-t} u_{i, \tau} \text {, we can rewrite } U_{i, t}^{B A U} \text { as: } \\
& U_{i, t}^{B A U}=\sum_{\tau=t}^{\infty} \delta^{\tau-t}\left[a \sum_{j \in N}\left(R_{j, \tau}+\frac{a}{b}\right)-\frac{b}{2}\left(\frac{a}{b}\right)^{2}-\frac{c}{2}\left(\frac{\delta}{1-\delta} \frac{a}{c}\right)^{2}\right] \\
& =\frac{a}{1-\delta} \sum_{j \in N} R_{j, t}+a \sum_{j \in N} \sum_{\tau=t}^{\infty} \frac{\delta^{\tau+1-t}}{1-\delta} y_{j, \tau}+\sum_{\tau=t}^{\infty} \delta^{\tau-t}\left[\left(n-\frac{1}{2}\right) \frac{a^{2}}{b}-\frac{c}{2}\left(\frac{\delta}{1-\delta} \frac{a}{c}\right)^{2}\right] \\
& =\frac{a}{1-\delta} \sum_{j \in N} R_{j, t}+\frac{a^{2}}{1-\delta}\left(n-\frac{1}{2}\right)\left(\frac{1}{b}+\frac{1}{c}\left[\frac{\delta}{1-\delta}\right]^{2}\right) \text {. }
\end{aligned}
$$

Similarly, the BAU payoff at time $T+1$ can be written as:

$$
U_{i, T+1}^{B}=\frac{a}{1-\delta} \sum_{j \in N}\left(R_{j, T+1}^{B A U}+Y_{j, T+1}\right)+\frac{a^{2}}{1-\delta}\left(n-\frac{1}{2}\right)\left(\frac{1}{b}+\frac{1}{c}\left[\frac{\delta}{1-\delta}\right]^{2}\right),
$$

where $Y_{i, T+1}$ measures the additional investments, relative to BAU, thanks to the first commitment period. Eeach party's present-discounted value of $Y_{i, T+1}$ is $a \frac{\delta^{T}}{1-\delta} \sum_{j} Y_{i, T+1}$, when evaluated in period 1. This term should be added when we derive the additional utility, relative to BAU, when the $n$ parties commit to $\mathbf{x}$ for $T$ periods at time $t=1$ (even if the parties thereafter returned to BAU). The additional utility, relative to BAU, is thus:

$$
\begin{aligned}
& \sum_{t=1}^{T} \delta^{t-1}\left[a \sum_{j \in N}\left(q_{j, t}^{B A U}+x_{j}\right)-\frac{b}{2}\left(q_{i, t}^{B A U}+x_{i}-R_{i, t}^{B A U}-Y_{i, t}\right)^{2}-\frac{c}{2}\left(r_{i, t}^{B}+y_{i, t}\right)^{2}-u_{i, t}^{B A U}\right] \\
& +a \frac{\delta^{T}}{1-\delta} \sum_{j \in N} Y_{j, T+1} \\
= & \sum_{t=1}^{T} \delta^{t-1}\left[a \sum_{j \neq i} x_{j}-\frac{b}{2}\left(x_{i}-Y_{i, t}\right)^{2}-\frac{c}{2} y_{i, t}^{2}+a Y_{i, t}-a \delta \frac{Y_{i, t+1}-Y_{i, t}}{1-\delta}\right]+a \delta^{T} \frac{\sum_{j \in N} Y_{j, T+1}}{1-\delta} \\
= & \sum_{t=1}^{T} \delta^{t-1}\left[a \sum_{j \neq i} x_{j}-\frac{b}{2}\left(x_{i}-Y_{i, t}\right)^{2}-\frac{c}{2} y_{i, t}^{2}\right]+a \frac{\delta^{T}}{1-\delta} \sum_{j \neq i} Y_{j, T+1},
\end{aligned}
$$

where the last equality follows because the three terms with $Y_{i, \tau}$ in (32) sum to zero for each $\tau=$ $\{2, \ldots, T+1\}$ and because $Y_{i, 1}=0$.

When the parties to not play BAU after the first commitment period, then, in order to obtain $i$ 's total additional payoff relative to BAU, we must add the additional payoff $\delta^{T} U_{i}\left(\mathbf{x}^{*}\right)$, where $U_{i}\left(\mathbf{x}^{*}\right)$ is the equilibrium additional utility relative to $\mathrm{BAU}$, in order to get $U_{i}(\mathbf{x})$ in Lemma 1. Q.E.D.

Proof of Lemma 2: Lemma 1 defines an optimal-control problem with control $y_{i, t}$. Note that the terminal value for $Y_{i, T+1}$ is zero because $U_{i}(\mathbf{x})$ is measured relative to $U_{i, 1}^{B A U}$ : this implies that $y_{i, T}=0$, i.e., the investment level in the final period coincides with the equilibrium investment level in BAU. In other words, there is no additional investment in the final period.

When $\lambda_{t}$ defines the shadow value of the stock $Y_{i, t}$, evaluated at time 1, the discrete-time Hamiltonian can be written as: ${ }^{33}$

$$
H_{t}=\delta^{t-1}\left[a \sum_{j \neq i} x_{j}-\frac{b}{2}\left(x_{i}-Y_{i, t}\right)^{2}-\frac{c}{2} y_{i, t}^{2}\right]+\lambda_{i, t+1}\left(Y_{i, t}+y_{i, t}\right),
$$

\footnotetext{
${ }^{33}$ I here apply Pontryagin's maximum principle for discrete time problems. For a general characterization and proof, see, for example, Leonard and van Long (1992:129-33).
} 
with first-order conditions

$$
y_{i, t}=\arg \max _{y_{i, t}} H_{t}=\lambda_{i, t+1} / c \delta^{t-1},
$$

adjoint equation

$$
\lambda_{i, t+1}-\lambda_{i, t}=-\frac{\partial H_{t}}{Y_{i, t}}=-\delta^{t-1} b\left(x_{i}-Y_{i, t}\right),
$$

and terminal condition

$$
\lambda_{i, T+1}=0 \Leftrightarrow y_{i, T}=0 .
$$

Combining the first two conditions and (8), we get the second-order difference equation:

$$
\begin{aligned}
c \delta^{t-2}\left(Y_{i, t}-Y_{i, t-1}\right)-c \delta^{t-1}\left(Y_{i, t+1}-Y_{i, t}\right) & =\delta^{t-1}\left(x_{i}-Y_{i, t}\right) b \Rightarrow \\
-Y_{i, t+1}+\left(\frac{1}{\delta}+1+\frac{b}{c}\right) Y_{i, t}-\frac{1}{\delta} Y_{i, t-1} & =x_{i} b / c,
\end{aligned}
$$

which has the solution (see, e.g., Sydsaeter and Hammon (1995:751-53)):

$$
\begin{aligned}
Y_{i, t} & =A_{1} m_{1}^{t-1}+A_{2} m_{2}^{t-1}+x_{i}, \text { where } \\
m_{1} & =\frac{1}{2}\left(\frac{1}{\delta}+1+\frac{b}{c}\right)-\frac{1}{2} \sqrt{\left(\frac{1}{\delta}+1+\frac{b}{c}\right)^{2}-\frac{4}{\delta}} \in(0,1), \\
m_{2} & =\frac{1}{2}\left(\frac{1}{\delta}+1+\frac{b}{c}\right)+\frac{1}{2} \sqrt{\left(\frac{1}{\delta}+1+\frac{b}{c}\right)^{2}-\frac{4}{\delta}}>1 .
\end{aligned}
$$

The constants $A_{1}$ and $A_{2}$ can be derived from the initial condition $Y_{i, 1}=0$, implying $A_{1}+A_{2}=-x_{i}$, and the terminal condition, $y_{i, T}=0$, implying

$$
\begin{aligned}
y_{i, T} & =Y_{i, T+1}-Y_{i, T}=A_{1} m_{1}^{T}\left(1-\frac{1}{m_{1}}\right)-\left(A_{1}+x_{i}\right) m_{2}^{T}\left(1-\frac{1}{m_{2}}\right)=0 \Rightarrow \\
A_{1} & =-\frac{m_{2}^{T}\left(1-\frac{1}{m_{2}}\right)}{m_{1}^{T}\left(\frac{1}{m_{1}}-1\right)+m_{2}^{T}\left(1-\frac{1}{m_{2}}\right)} x_{i}, \text { and } \\
A_{2} & =-A_{1}-x_{i}=\frac{m_{2}^{T}\left(1-\frac{1}{m_{2}}\right)}{m_{1}^{T}\left(\frac{1}{m_{1}}-1\right)+m_{2}^{T}\left(1-\frac{1}{m_{2}}\right)} x_{i}-x_{i}=-\frac{m_{1}^{T}\left(\frac{1}{m_{1}}-1\right)}{m_{1}^{T}\left(\frac{1}{m_{1}}-1\right)+m_{2}^{T}\left(1-\frac{1}{m_{2}}\right)} x_{i} .
\end{aligned}
$$

With the definitions $k_{1}=-A_{1} x_{i}$ and $k_{2}=-A_{2} x_{i}$, (33) can be written as in Lemma 2. Q.E.D.

Proof of Lemma 3: By substituting in for $y_{i, t}$ and $Y_{i, t}$ into $U_{i, 1}(\mathbf{x})$, defined in Lemma 1, we get:

$$
\begin{aligned}
& U_{i}(\mathbf{x})-\delta^{T} U_{i}\left(\mathbf{x}^{*}\right)=\sum_{t=1}^{T} \delta^{t-1}\left[a \sum_{j \neq i} x_{j}-\frac{b}{2}\left(x_{i}-Y_{i, t}\right)^{2}-\frac{c}{2} y_{i, t}^{2}\right]+a \frac{\delta^{T}}{1-\delta} \sum_{j \neq i} Y_{j, T+1} \\
= & \sum_{t=1}^{T} \delta^{t-1}\left[a \sum_{j \neq i} x_{j}-\frac{b}{2} x_{i}^{2}\left(k_{1} m_{1}^{t-1}+k_{2} m_{2}^{t-1}\right)^{2}-\frac{c}{2}\left[x_{i}\left(k_{1} m_{1}^{t-1}\left[1-m_{1}\right]-k_{2} m_{2}^{t-1}\left[m_{2}-1\right]\right)\right]^{2}\right] \\
& +a \frac{\delta^{T}}{1-\delta} \sum_{j \neq i} Y_{j, T+1} \\
= & \alpha \sum_{j \neq i} x_{j}+\beta x_{i}^{2} / 2, \text { if just } \\
& \alpha \equiv \sum_{t=1}^{T} \delta^{t-1} a+a \frac{\delta^{T}}{1-\delta} \frac{Y_{j, T+1}}{x_{j}}=\frac{a}{1-\delta}\left[1-\delta^{T}\left(k_{1} m_{1}^{T-1}+k_{2} m_{2}^{T-1}\right)\right] \text { and } \\
& \beta \equiv \sum_{t=1}^{T} \delta^{t-1}\left[b\left(k_{1} m_{1}^{t-1}+k_{2} m_{2}^{t-1}\right)^{2}+c\left[\left(k_{1} m_{1}^{t-1}\left[1-m_{1}\right]-k_{2} m_{2}^{t-1}\left[m_{2}-1\right]\right)\right]^{2}\right] . \text { Q.E.D. }
\end{aligned}
$$


Proof of Propositions 1-3: The proof of Proposition 1 follows from the earlier Lemmata, while Propositions 2 and 3 follow from the reasoning in the text.

Proof of Proposition 4: If $i$ defects by not contributing at time $t$, then $i$ can still benefit $a \sum_{j \neq i} x_{j}+\frac{a \delta}{1-\delta} \sum_{j \neq i} y_{j, t}$, since $j$ 's investments will raise $j$ 's contribution in the future, even when the parties return to BAU. This benefit is largest at $t=1$, since $y_{j, t}$ is decreasing in $t \in\{1, \ldots, T\}$, as noticed already.

When defection is punished by a reversion to BAU for $l \leq \infty$ periods with probability $\phi \in(0,1]$, then compliance (giving payoff $U_{i}^{*}$ ) is better at time $t=1$ if:

$$
a \sum_{j \neq i} x_{j}+\frac{a \delta}{1-\delta} \sum_{j \neq i} y_{j, 1}+\delta\left(1-\phi+\phi \delta^{l}\right) U_{i}^{*} \leq U_{i}^{*} .
$$

When we substitute in for $y_{j, 1}, x_{j}^{*}$, and $U_{i}^{*}$, this inequality becomes:

$$
\begin{aligned}
a\left(\sum_{j \neq i} x_{j}^{*}+\frac{\delta}{1-\delta} \sum_{j \neq i} y_{j, 1}^{*}\right) & \leq\left[1-\delta\left(1-\phi+\phi \delta^{l}\right)\right] U_{i}^{*} \Rightarrow \\
a\left[1+\frac{\delta}{1-\delta}\left(1-k_{1} m_{1}-k_{2} m_{2}\right)\right] \sum_{j \neq i} x_{j}^{*} & \leq\left[1-\delta\left(1-\phi+\phi \delta^{l}\right)\right] \frac{\alpha^{2}(n-1)^{2}}{\beta\left(1-\delta^{T}\right)} w\left(1-\frac{w}{2}\right) \Rightarrow \\
\frac{a\left(1-\delta^{T}\right)}{\alpha\left[1-\delta\left(1-\phi+\phi \delta^{l}\right)\right]}\left[\frac{1-\delta\left(k_{1} m_{1}+k_{2} m_{2}\right)}{1-\delta}\right] & \leq 1-\frac{w}{2} \Rightarrow \\
w & \leq 2-2 \frac{1-\delta\left(k_{1} m_{1}+k_{2} m_{2}\right)}{(1-\delta)\left[1-\delta\left(1-\phi+\phi \delta^{l}\right)\right]} \frac{a\left(1-\delta^{T}\right)}{\alpha},
\end{aligned}
$$

which equals (16) when $\phi=1$ and $l \rightarrow \infty$. Q.E.D.

Proof of Proposition 5: Proposition 5 follows straightforwardly from the equilibrium continuation values, derived above.

Proof of Proposition 6:

(i) Contracts on investments: I will first permit the negotiated $\mathbf{y}_{t}=\left(y_{1, t}, \ldots, y_{n, t}\right)$ to be timedependent, so that $\mathbf{y}=\left(\mathbf{y}_{1}, \ldots, \mathbf{y}_{T}\right)$ is a matrix. Lemma 1 presents a reformulation of the problem and (when we remove the max-operator) it holds regardless of how the $x_{i, t}$ 's and the $y_{i, t}$ 's are decided on. When $y_{i, t}$ is committed to, but not $x_{i, t}$, the latter follows straightforwardly from $i$ 's maximization problem and, just as in BAU,

$$
q_{i, t}-R_{i, t}=a / b \Rightarrow x_{i}=Y_{i, t} .
$$

The continuation value can thus be written as a function of the investments matrix $\mathbf{y}$ :

$$
\begin{aligned}
U_{i}(\mathbf{y}) & =\sum_{t=1}^{T} \delta^{t-1}\left[a \sum_{j \neq i} \sum_{t^{\prime}=1}^{t-1} y_{j, t^{\prime}}-\frac{c}{2} y_{i, t}^{2}\right]+a \frac{\delta^{T}}{1-\delta} \sum_{j \neq i} \sum_{t^{\prime}=1}^{T} y_{j, t^{\prime}}+\delta^{T} U_{i}\left(\mathbf{y}^{*}\right) \Rightarrow \\
U_{i}(\mathbf{y})-\delta^{T} U_{i}\left(\mathbf{y}^{*}\right) & =\sum_{t=1}^{T}\left[\alpha_{t} \sum_{j \neq i} y_{j, t}-\frac{\beta_{t}}{2} y_{i, t}^{2}\right], \text { where } \alpha_{t}=\frac{a \delta^{t}}{1-\delta} \text { and } \beta_{t}=\delta^{t-1} c .
\end{aligned}
$$

If we require a time-independent $y_{j, t}=y_{j}$, we can write

$$
U_{i}(\mathbf{y})-\delta^{T} U_{i}\left(\mathbf{y}^{*}\right)=\alpha \sum_{j \neq i} y_{j}-\frac{\beta}{2} y_{i}^{2}, \text { where } \alpha=\delta a \frac{1-\delta^{T}}{(1-\delta)^{2}} \text { and } \beta=\sum_{t=1}^{T} \delta^{t-1} c=c \frac{1-\delta^{T}}{1-\delta} .
$$


Just as before, we can write $i$ 's payoff as in Example E. Consequently, the proofs for the other propositions follow the same steps as above. Proposition 1 gives, for example:

$$
y_{j}^{*}=w(n-1) \alpha / \beta=w(n-1) \frac{\delta a / c}{1-\delta} .
$$

Time-dependent investment levels: Since $i$ 's payoff is separable in the $y_{j, t}$ 's, we can apply Corollary 1 for each $\mathbf{y}_{t}$, if we fix the investment levels for the other periods, in order to get:

$$
y_{j, t}^{*}=w(n-1) \alpha_{t} / \beta_{t}=w(n-1) \frac{\delta a / c}{1-\delta},
$$

which equals $y_{j}^{*}$. Hence, the restriction to time-independent investment levels is nonbinding: the equilibrium is the same in both cases.

The choice of $T$ is also irrelevant in both cases, since the equilibrium continuation value is:

$$
U_{i}\left(\mathbf{y}^{*}\right)=\delta a \frac{1}{(1-\delta)^{2}}(n-1)^{2} w \frac{\delta a / c}{1-\delta}-\frac{c / 2}{1-\delta}\left[(n-1) w \frac{\delta a / c}{1-\delta}\right]^{2}=\frac{[\delta a(n-1)]^{2}}{c(1-\delta)^{3}} w\left(1-\frac{w}{2}\right) .
$$

(ii) Contracts on carbon tax: I will first permit $z_{t}=\left(z_{1, t}, \ldots, z_{n, t}\right)$ to be time-dependent, so that $z=\left(\mathbf{z}_{1}, \ldots, \mathbf{z}_{T}\right)$ is a matrix.

With an emission tax equal to $z_{i, t}$, collected by the government in country $i$, the equilibrium ensures that the marginal benefit when consuming fossil fuel (or the marginal abatement cost) equals $z_{i, t}$. This implies:

$$
x_{i, t}-Y_{i, t}=z_{i, t} / b,
$$

and, therefore, $i$ 's continuation value can be written as the function

$$
U_{i}(\mathbf{z})=\sum_{t=1}^{T} \delta^{t-1}\left[a \sum_{j \neq i}\left(z_{j, t} / b+Y_{j, t}\right)-\frac{z_{i, t}^{2}}{2 b}-\frac{c}{2} y_{i, t}^{2}\right]+a \frac{\delta^{T}}{1-\delta} \sum_{j \neq i} Y_{j, T+1}+\delta^{T} U_{i}\left(\mathbf{z}^{*}\right)
$$

so, there is no value for $i$ to invest beyond the BAU-levels, and $y_{i, t}=0$, so:

$$
\begin{aligned}
U_{i}(\mathbf{z})-\delta^{T} U_{i}\left(\mathbf{z}^{*}\right) & \equiv \sum_{t=1}^{T} \delta^{t-1}\left[a \sum_{j \neq i} z_{j, t} / b-\frac{z_{i, t}^{2}}{2 b}\right]=\sum_{t=1}^{T}\left[\alpha_{t} \sum_{j \neq i} z_{j, t}-\frac{\beta_{t}}{2} z_{i, t}^{2}\right], \text { where } \\
\alpha_{t} & =a \delta^{t-1} / b \text { and } \beta_{t}=\delta^{t-1} / b .
\end{aligned}
$$

If the emission tax is time-independent, we can write:

$$
U_{i}(\mathbf{z})-\delta^{T} U_{i}\left(\mathbf{z}^{*}\right)=\alpha \sum_{j \neq i} z_{j}-\frac{\beta}{2} z_{i}^{2}, \text { where } \alpha=\frac{a}{b} \frac{1-\delta^{T}}{1-\delta} \text { and } \beta=\frac{1}{b} \frac{1-\delta^{T}}{1-\delta} .
$$

In this case, Corollary 1 implies:

$$
z_{i}^{*}=w(n-1) \alpha / \beta=w(n-1) a .
$$

Time-dependent tax: Since $i$ 's payoff is separable in the $z_{j, t}$ 's, we can apply Corollary 1 for each $z_{t}$, if we fix the emission tax levels for the other periods, in order to get:

$$
z_{j, t}^{*}=w(n-1) \alpha_{t} / \beta_{t}=w(n-1) a,
$$

which equals $z_{i}^{*}$. Hence, the restriction to time-independent emission tax levels is nonbinding: the equilibrium is the same in both cases.

The choice of $T$ is also irrelevant in both cases, since the equilibrium continuation value is:

$$
U_{i}\left(\mathbf{z}^{*}\right)=\frac{a}{b} \frac{1}{1-\delta}(n-1)^{2} w a-\frac{1}{2} \frac{1}{b} \frac{1}{1-\delta}[(n-1) w a]^{2}=\frac{[a(n-1)]^{2}}{b(1-\delta)} w\left(1-\frac{w}{2}\right) .
$$


By comparison, a tax gives higher payoff than an investment agreements if:

$$
\frac{[a(n-1)]^{2}}{b(1-\delta)}>\frac{[\delta a(n-1)]^{2}}{c(1-\delta)^{3}} \Rightarrow c(1-\delta)^{2}>b \delta^{2} \Rightarrow \frac{1}{\delta}>1+\sqrt{\frac{b}{c}} .
$$

Clearly, the investment agreement is better if investments are inexpensive and the tax ineffective (because $b$ is large). If $\delta$ is large, investments are, in effect, less expensive, and thus the investment agreement is more attractive.

(iii) Combining (i) and (ii): When the parties face both a matrix of emission taxes and a matrix of investment levels, $i$ 's payoff can be written as:

$$
\begin{aligned}
& U_{i}(\mathbf{x})-\delta^{T} U_{i}\left(\mathbf{x}^{*}\right) \equiv \sum_{t=1}^{T} \delta^{t-1}\left[a \sum_{j \neq i}\left(\frac{z_{j, t}}{b}+Y_{j, t}\right)-\frac{z_{j, t}^{2}}{2 b}-\frac{c}{2} y_{i, t}^{2}\right]+a \frac{\delta^{T}}{1-\delta} \sum_{j \neq i} Y_{j, T+1} \\
= & {\left[\sum_{t=1}^{T} \delta^{t-1}\left(a \sum_{j \neq i} Y_{j, t}-\frac{c}{2} y_{i, t}^{2}\right)+a \frac{\delta^{T}}{1-\delta} \sum_{j \neq i} Y_{j, T+1}\right]+\left[\sum_{t=1}^{T} \delta^{t-1}\left(a \sum_{j \neq i} \frac{z_{j, t}}{b}-\frac{z_{j, t}^{2}}{2 b}\right)\right], }
\end{aligned}
$$

where the first (second) bracket can be recognized as $i$ 's payoff in the situation when only the investment levels (the emission taxes) were negotiated. The two problems are thus separable, and the results above continue to hold when the parties can negotiate both policy instruments. In this case, the additional payoff, relative to BAU, is also the sum of the two additional payoffs, derived above:

$$
U_{i}\left(\mathbf{y}^{*}\right)+U_{i}\left(\mathbf{z}^{*}\right)=\left[\frac{1}{c(1 / \delta-1)^{2}}+\frac{1}{b}\right] \frac{[a(n-1)]^{2}}{(1-\delta)} w\left(1-\frac{w}{2}\right) .
$$

(iv) Complete contracts: When the parties negotiate the investment levels, the $z_{j, t}$ 's pin down the $x_{j, t}$ 's, given the $y_{j, t}$ 's, so negotiating the $z_{j, t}$ 's is then equivalent to negotiating the $x_{j, t}$ 's: Also when the $y_{j, t}$ 's and the $x_{j, t}$ 's are negotiated, the contract is complete and the choice of $T$ is irrelevant. One optimal $T$ is thus $T=\infty$.

(v) Time-path for $x$ : When the $y_{j, t}$ 's and the $x_{j, t}$ 's are negotiated, one optimal $T$ is $T=\infty$. In this situation, pinning down the $x_{j, t}$ 's is equivalent to pinning down both the $y_{j, t}$ 's and the $x_{j, t}$ 's, because there is no externality when it comes to the $y_{j, t}$ 's (given every future $x_{j, t}$ ) and, hence, every party will invest optimally, without any need to specify the investment levels.

This reasoning completes the proof but, to illustrate, consider the time profile for the contribution levels when the parties negotiate both the emission taxes and the investment levels:

$$
x_{i, t}=(n-1) w a / b+t(n-1) w \frac{\delta a / c}{1-\delta} .
$$

Given this path, optimal investments, from $i$ 's point of view, are:

$$
\begin{aligned}
c y_{i, t-1}-\delta c y_{i, t} & =\delta b\left(x_{i, t}-Y_{i, t}\right)=\delta b\left((n-1) w a / b+t(n-1) w \frac{\delta a / c}{1-\delta}-t(n-1) w \frac{\delta a / c}{1-\delta}\right) \\
& =\delta b((n-1) w a / b) \Rightarrow y_{i, t-1}=\frac{\delta(n-1) w a}{c(1-\delta)},
\end{aligned}
$$

just as in the optimal contract. So, the combination of negotiating investment levels and emission taxes is indeed equivalent to pinning down the path of $x_{i, t}$.

(vi) Firms: It suffices to prove that when $T=1$ and the parties negotiate $x_{i, t}$ at the start of every period $t$, and the firms invest to maximize profit, then the outcome coincides with the outcome when all the $y_{i, t}$ 's and the $x_{i, t}$ 's are negotiated at the very beginning.

When only this period's $x_{i, t}$ are negotiated at the start of period $t$, then, when applying Corollary 1 :

$$
b\left(x_{i, t}-Y_{i, t}\right)=a w(n-1) .
$$


Firms invest such as to equalize the marginal investment cost to the present-discounted value of their investment, where the willingness to pay for more $R_{i, t}$ equals $b\left(q_{i, t}-R_{i, t}\right)$ at time $t$. Thus:

$$
\begin{aligned}
c r_{i, t} & =\sum_{t=1}^{\infty} \delta^{t} b\left(q_{i, t}-R_{i, t}\right)=\sum_{t=1}^{\infty} \delta^{t} b\left(q_{i, t}^{B A U}-R_{i, t}^{B A U}+x_{i, t}-Y_{i, t}\right) \\
& =\sum_{t=1}^{\infty} \delta^{t} b\left(\frac{a}{b}+x_{i, t}-Y_{i, t}\right)=\sum_{t=1}^{\infty} \delta^{t} b\left(\frac{a}{b}+\frac{a}{b} w(n-1)\right)=\frac{\delta}{1-\delta} b\left(\frac{a}{b}+\frac{a}{b} w(n-1)\right) .
\end{aligned}
$$

With $r_{i, t}=r_{i, t}^{B A U}+y_{i, t}$ and $r_{i, t}^{B A U}=\frac{\delta}{1-\delta} \frac{a}{c}$, it follows that $c y_{i, t}=\frac{\delta}{1-\delta} a w(n-1)$, just as with complete contracts.

(vii)-(ix) are trivial and thus omitted.

(x) Compliance: In all the above situations, and also in the basic model if $c \rightarrow \infty$, we have that $U_{i}^{*}$ is independent of $T$ and it can, when the policy instrument is given by the matrix $\psi=\left(\boldsymbol{\psi}_{1}, \ldots, \boldsymbol{\psi}_{K}\right)$, where $\psi_{k}=\left(\psi_{1, k}, \ldots \psi_{n, k}\right)$ for each $k \in\{1, \ldots, K\}$, be written as the following (for some constants $\alpha_{k}$ and $\beta_{k}$ ):

$$
U_{i}^{*}=\sum_{k \in\{1, \ldots, K\}} \frac{1}{1-\delta}\left[\alpha_{k}^{\prime} \sum_{j \neq i} \psi_{j, k}-\frac{\beta_{k}^{\prime}}{2} \psi_{j, k}^{2}\right], \text { so } \psi_{j, k}=w(n-1) \alpha_{k}^{\prime} / \beta_{k}^{\prime},
$$

from Corollary 1. If defection is punished by reverting to BAU for $l$ periods with probability $\phi$, then the incentive constraint is:

$$
\begin{aligned}
& \sum_{k \in\{1, \ldots, K\}} \alpha_{k}^{\prime} \sum_{j \neq i} \psi_{j, k}+\delta\left(1-\phi+\phi \delta^{l}\right) U_{i}^{*} \leq U_{i}^{*} \Rightarrow \\
& \sum_{k \in\{1, \ldots, K\}} \frac{(n-1)^{2}\left(\alpha_{k}^{\prime}\right)^{2}}{\beta_{k}^{\prime}} w \leq \sum_{k \in\{1, \ldots, K\}} \frac{1-\delta\left(1-\phi+\phi \delta^{l}\right)}{1-\delta}\left[\frac{(n-1)^{2}\left(\alpha_{k}^{\prime}\right)^{2}}{\beta_{k}^{\prime}} w-\frac{\beta_{k}^{\prime}}{2}\left[\frac{(n-1) \alpha_{k}^{\prime}}{\beta_{k}^{\prime}} w\right]^{2}\right] \Rightarrow \\
& 1 \leq \frac{1-\delta\left(1-\phi+\phi \delta^{l}\right)}{1-\delta}\left[1-\frac{1}{2} w\right] \Rightarrow \\
& w \leq 2-2 \frac{1-\delta}{1-\delta\left(1-\phi+\phi \delta^{l}\right)}=2 \frac{1-\delta\left(1-\phi+\phi \delta^{l}\right)-1+\delta}{1-\delta\left(1-\phi+\phi \delta^{l}\right)}=2 \delta \frac{\phi\left(1-\delta^{l}\right)}{1-\delta\left(1-\phi+\phi \delta^{l}\right)},
\end{aligned}
$$

which simplifies to $w \leq 2 \delta$ if $\phi=1$ and $l=\infty$. Q.E.D. 


\section{REFERENCES}

Abreu, D., and F. Gul (2000): "Bargaining and Reputation," Econometrica 68(1): 85-117.

Abreu, D., and D. Pearce (2007): "Bargaining, Reputation, and Equilibrium Selection in Repeated Games with Contracts," Econometrica 75(3): 653-710.

Abreu, D., and D. Pearce (2015): "A Dynamic Reinterpretation of Nash Bargaining With Endogenous Threats," Econometrica 83(4): 1641-55.

Acemoglu, D., P. Aghion, L. Bursztyn, and D. Hemous (2012): "The Environment and Directed Technical Change," American Economic Review 102(1):131-66.

Aldy, J. E., S. Barrett, and R. N. Stavins (2003): "Thirteen plus one: a comparison of global climate policy architectures," Climate Policy 3(4): 373-97.

Andersson, O., C. Argenton, and J. W. Weibull (2018): "Robustness to Strategic Uncertainty in the Nash Demand Game," Mathematical Social Sciences 91:1-5.

Asheim, G. (1992): "A Unique Solution to n-Person Sequential Bargaining," Games and Economic Behavior 4: 169-81.

Bagwell, K., R. W. Staiger, and A. Yurukoglu (2018): "Quantitative Analysis of Multi-Party Tariff Negotiations," mimeo, Stanford University.

Barrett, S. (1994): "Self-enforcing international environmental agreements," Oxford Economic Papers, 46: 878-94.

Barrett, S. (2002): "Consensus Treaties," Journal of Institutional and Theoretical Economics 158(4): 529-47.

Barrett, S., and A. Dannenberg (2016): "An experimental investigation into 'pledge and review' in climate negotiations," Climatic Change 138(1): 339-51.

Battaglini, M., and B. Harstad (2016): "Participation and Duration of Environmental Agreements," Journal of Political Economy 124(1): 160-204.

Beccherle, J., and J. Tirole (2011): "Regional Initiatives and the Cost of Delaying Binding Climate Change Agreements," Journal of Public Economics 95: 1339-48.

Bernauer, T., A. Kalbhenn, V. Koubi, and G. Spilker (2013): "Is there a 'Depth versus Participation' Dilemma in International Cooperation?" Review of International Organization 8 (4): 477-97.

Binmore, K. (1987): "Nash Bargaining Theory (II)," The Economics of Bargaining, ed. by K. Binmore and P. Dasgupta (ed.). Cambridge: Basil Blackwell.

Binmore, K., M. J. Osborne, and A. Rubinstein (1992): "Non-Cooperative Models of Bargaining," Handbook of Game Theory, Volume 1, ed. by R. J. Aumann and S. Hart, Elsevier Science Publishers.

Binmore, K., A. Rubinstein, and A. Wolinsky (1986): "The Nash Bargaining Solutin in Economic Modelling," The RAND Journal of Economics 17(2): 176-88.

Bloch, F. (2018): "Coalitions and networks in oligopolies," Handbook of Game Theory and Industrial Organization, ed. by. L. Corchon and M. Marini, Edward Elgar.

Bodansky, D. (2010): "The Copenhagen Climate Change Conference: A Postmortem," American Journal of International Law 104(2): 230-40.

Bodansky, D., J. Brunnee, and L. Rajamani (2017): International Climate Change Law, Oxford University Press.

Bodansky, D., and L. Rajamani (2017): "Evolution and Governance Architecture of the Climate Change Regime," forthcoming in Global Climate Policy: Actors, Concepts, and Enduring Challenges, ed. by U. Luterbacher and D. Sprinz, MIT Press.

Britz, V., P. J. Herings, and A. Predtetchinski (2010): "Non-cooperative Support for the Asymmetric Nash Bargaining Solution," Journal of Economic Theory 145: 1951-67.

Bulow, J., and P. Klemperer (1996): "Auctions Versus Negotiations," American Economic Review 86(1): 180-94.

Bulow, J., and P. Klemperer (2009): "Why Do Sellers (Usually) Prefer Auctions?" American Economic Review 99(4): 1544-75.

Calvo, E., and S. J. Rubio (2012): "Dynamic Models of International Environmental Agreements: A Differential Game Approach," International Review of Environmental and Resource Economics 6: 289339. 
Caparrós, A. (2016): "Bargaining and International Environmental Agreements," Environmental and Resource Economics 65(1): 5-31.

Carlsson, H. (1991): "A Bargaining Model Where Parties Make Errors," Econometrica 59(5): 1487-96.

Carraro, C., and D. Siniscalco (1993): "Strategies for the International Protection of the Environment." Journal of Public Econonomics 52(3): 309-28.

Chae, S., and J. Yang (1994): "An N-person pure bargaining game", Journal of Economic Theory 62(1): $86-102$.

Cho, I., and A. Matsui (2013): "Search Theory, Competitive Equilibrium and the Nash Bargaining Solution," Journal of Economic Theory 148(4): 1659-88.

Compte, O., and P. Jehiel (2010): "Bargaining and Majority Rules: A Collective Search Perspective," Journal of Political Economy 118(2): 189-221.

d'Aspremont, C., A. Jacquemin, J. J. Gabszewicz, and J. A. Weymark (1983): "On the stability of collusive price leadership," The Canadian Journal of Economics 16(1): 17-25.

Dutta, P. K., and R. Radner (2004): "Self-enforcing climate-change treaties," Proceedings of the National Academy of Science 101: 4746-51.

Dutta, P. K., and R. Radner (2006): "A Game-Theoretic Approach to Global Warming," Advances in Mathematical Economics 8: 135-53.

Dutta, P. K., and R. Radner (2018): "The Paris Accord Can Be Effective if the Green Climate Fund is Effective," mimeo, Columbia University.

Finus, M., and S. Maus (2008): "Modesty may pay!" Journal of Public Economic Theory 10: 801-26.

Gilligan, M. J. (2004): "Is There a Broader-Deeper Trade-off in International Multilateral Agreements?" International Organization 58 (3): 459-84.

Gollier, C., and J. Tirole (2015): "Making Climate Agreements Work," The Economist, guest blog, June 1st: https://www.economist.com/free-exchange/2015/06/01/making-climate-agreements-work

Golosov, M., J. Hassler, P. Krusell, and A. Tsyvinski (2014): "Optimal Taxes on Fossil Fuel in General Equilibrium," Econometrica 82(1): 41-88.

Harris, M., and B. Holmstrom (1987): "On The Duration of Agreements," International Economic Review 28(2): 389-406.

Harsanyi, J., and R. Selten (1972): "A Generalized Nash Solution for Two-Person Bargaining Games with Incomplete Information," Management Science 18(5) part 2: 80-106.

Harstad, B. (2012): "Climate Contracts: A Game of Emissions, Investments, Negotiations, and Renegotiations," Review of Economic Studies 79(4): 1527-57.

Harstad, B. (2016): "The Dynamics of Climate Agreements," Journal of the European Economic Association 14(3): 719-52.

Harstad, B., F. Lancia, and A. Russo (2018): "Compliance Technology and Self-Enforcing Agreements," mimeo, University of Oslo.

Hoel, M. (1992): "International environmental conventions: the case of uniform reductions of emissions," Environmental and Resource Economics 2(2): 141-59.

Howard, J. V. (1992): "A social choice rule and its implementation in perfect equilibrium," Journal of Economic Theory 56(1): 142-59.

IPCC (2014): Climate Change 2014: Mitigation of Climate Change. Contribution of Working Group III to the Fifth Assessment Report of the Intergovernmental Panel on Climate Change. Cambridge University Press.

Kalai, E. (1977): "Non-symmetric Nash Solutions and Replication of 2-Person Bargaining," International Journal of Game Theory 6(3): 129-33.

Kambe, S. (2000): "Bargaining with Imperfect Commitment," Games and Economic Behavior 28: 21737.

Keohane, R. O., and M. Oppenheimer (2016): "Paris: Beyond the Climate Dead End through Pledge and Review," Politics and Governance 4(3): 142-51.

Kolstad, C. D., and M. Toman (2005): "The Economics of Climate Policy," Handbook of Environmental Economics 3: 1562-93.

Krishna, V., and R. Serrano (1996): "Multilateral Bargaining", Review of Economic Studies 63(1): 61-80.

Laurelle, A., and F. Valenciano (2008): "Non-Cooperative Foundations of Bargaining Power in Committees and the Shapley-Shubik Index," Games and Economic Behavior 63: 341-53. 
Leonard, D., and N. Van Long (1992): Optimal Control Theory and Static Optimization in Economics, Cambridge University Press.

Martimort, D., and W. Sand-Zantman (2016): "A Mechanism Design Approach to Climate-change Agreements," Journal of the European Economic Association 14(3): 669-718.

McAfee, P. (1993): "Mechanism Design by Competing Sellers," Econometrica 61(6): 1281-1312.

Miyakawa, T. (2008): "Note on the Equal Split Solution in an n-Person Non-Cooperative Bargaining Game," Mathematical Social Sciences 55(3): 281-91.

Myerson, R. (1978): "Refinement of the Nash Equilibrium Concept," International Journal of Game Theory 7: 73-80.

Nash, J. (1950): "The Bargaining Problem," Econometrica 18: 155-62.

Nash, J. (1953): "Two-Person Cooperative Games," Econometrica 21(1): 128-40.

Nordhaus, W. D. (2015): "Climate Clubs: Overcoming Free-riding in International Climate Policy," American Economic Review 105(4): 1339-70.

OECD (2018): "Common time frames: Summary of discussions at the March 2018 Climate Change Expert Group Global Forum," Note prepared by the OECD/IEA Climate Change Expert Group.

Osborne, M. J., and A. Rubinstein (1990): Bargaining and Markets, Academic Press.

Roth, A. (1979): "Proportional Solutions to the Bargaining Problem," Econometrica 47(3): 775-78.

Rubinstein, A. (1982): "Perfect Equilibrium in a Bargaining Model," Econometrica 50(1): 97-109.

Schmalensee, R. (1998): "Greenhouse policy architectures and institutions," Economics and Policy Issues in Climate Change, ed. by W. D. Nordhaus, Resources for the Future Press, Washington, D. C.

Segal, I. (1999): "Complexity and Renegotiation: A Foundation for Incomplete Contracts," Review of Economic Studies 66(1): 57-82.

Selten, R. (1975): "Reexamination of the Perfectness Concept for Equilibrium Points in Extensive Games," International Journal of Game Theory 4: 25-55.

Simon, L. K. (1987): "Local Perfection," Journal of Economic Theory 43: 134-56.

Simon, L. K., and M. B. Stinchcombe (1995): "Equilibrium Refinement for Infinite Normal-Form Games," Econometrica 63(6): 1421-43.

Stern, N. (2006): The Economics of Climate Change: The Stern Review, Cambridge University Press

Sutton, J. (1986): "Non-Cooperative Bargaining Theory: An Introduction," The Review of Economic Studies 53(5): 709-24.

Sydsaeter, K., and P. J. Hammond (1995): Mathematics for Economic Analysis, Prentice Hall.

Victor, D. (2015): "Why Paris Worked: A Different Approach to Climate Diplomacy," Yale Envir. 360: https://e360.yale.edu/features/why_paris_worked_a_different_approach_to_climate_diplomacy 